UWThPh-2011-2

MPP-2011-8cd

\title{
Ultrasoft NLL Running of the Nonrelativistic $O(v)$ QCD Quark Potential
}

\author{
André H. Hoang*1,2 and Maximilian Stahlhofen ${ }^{\dagger 1,3}$ \\ ${ }^{1}$ University of Vienna, \\ Faculty of Physics, Boltzmanngasse 5, \\ A-1090 Wien, Austria \\ ${ }^{2}$ Max-Planck-Institut für Physik, \\ Föhringer Ring 6, \\ 80805 München, Germany \\ ${ }^{3}$ Grup de Física Teòrica and IFAE, \\ Universitat Autònoma de Barcelona, \\ E-08193 Bellaterra, Barcelona, Spain
}

\begin{abstract}
Using the nonrelativistic effective field theory vNRQCD, we determine the contribution to the next-to-leading logarithmic (NLL) running of the effective quark-antiquark potential at order $v$ $(1 / m|\mathbf{k}|)$ from diagrams with one potential and two ultrasoft loops, $v$ being the velocity of the quarks in the c.m. frame. The results are numerically important and complete the description of ultrasoft next-to-next-to-leading logarithmic (NNLL) order effects in heavy quark pair production and annihilation close to threshold.
\end{abstract}

\footnotetext{
* Electronic address: Andre.Hoang@univie.ac.at

$\dagger$ Electronic address: Maximilian.Stahlhofen@univie.ac.at
} 


\section{INTRODUCTION}

The measurement of the line-shape of the total top-antitop quark production cross section in the threshold region $\sqrt{s} \approx 2 m_{t}$ is one of the major tasks of the top quark physics program at a future linear collider. The most prominent quantity to be measured is the top quark mass, and one can expect an improvement in precision of $m_{t}$ by about an order of magnitude to mass measurements based on reconstruction obtained at the Tevatron and the LHC [1]. In addition, fitting the lineshape measurements to theoretical higher order predictions allows to control precisely the top mass scheme, which is not the case for mass reconstruction at hadron colliders based on Monte-Carlo generators.

To obtain a meaningful theoretical description of the nonrelativistic threshold dynamics it is required to systematically sum the so-called Coulomb singular terms $\propto\left(\alpha_{s} / v\right)^{n}$ in a systematic nonrelativistic expansion, $v$ being the relative velocity of the top quarks. This task is achieved by means of effective theories based on nonrelativistic QCD (NRQCD) [2]. In this approach, however, sizeable logarithmic terms $\propto\left(\alpha_{s} \ln v\right)^{n}$ are not systematically accounted for, which leads to rather large normalization uncertainties of the cross section line-shape. The next-to-next-to-leading order (NNLO) predictions in this "fixed-order" approach were estimated to have a normalization uncertainty of order 20\% [3]. Presently known NNNLO fixed-order corrections, e.g. [4-7], seem to reduce this uncertainty to about $10 \%$ [8]. This normalization uncertainty might not affect the top mass measurement, which primarily depends on the c.m. energy where the cross section rises, but it still renders precision measurements of other quantities such as the top total width or the top quark couplings impossible. On the other hand, such large normalization uncertainties might cast general doubts on the reliability of the theoretical method itself. To match the statistical uncertainties, that are expected for these quantities at an International Linear Collider (ILC), a theoretical precision of the cross section normalization of at least $3 \%$ would be required.

In Refs. [9] (see also Ref. [10]) it was demonstrated that the summation of logarithmic $\left(\alpha_{s} \ln v\right)^{n}$ terms, using renormalization-group-improved (RGI) perturbation theory, can significantly reduce the normalization uncertainties of the threshold cross section. Concerning QCD effects the RGI leading-logarithmic (LL) and NLL order predictions of the threshold cross section are completely known, but no full NNLL order prediction exists at present. The full NNLL order prediction is, however, required to obtain a reliable estimate of the remaining theoretical normalization uncertainties.

The missing ingredient is the NNLL running of the Wilson coefficient of the leading order effective current that describes production and annihilation of a nonrelativistic $t \bar{t}$ pair in a S-wave spin-triplet state $\left({ }^{3} S_{1}\right)$. Adopting the label notation from vNRQCD [11, 13] the current has the form

$$
\mathbf{J}_{1, \mathbf{p}}=\psi_{\mathbf{p}}^{\dagger} \boldsymbol{\sigma}\left(i \sigma_{2}\right) \chi_{-\mathbf{p}}^{*}
$$

where $\psi_{\mathbf{p}}$ and $\chi_{\mathbf{p}}$ annihilate top and antitop quarks with three-momentum $\mathbf{p}$, respectively, and where color indices have been suppressed. The current does not have a LL anomalous dimension because there is no one-loop vertex diagram in the effective theory that contains UV divergences associated with the current $\mathbf{J}_{1, \mathbf{p}}$. Such UV divergences arise at NLL order from insertions of the next-to-next-to-leading kinetic energy operators and from insertions of the next-to-next-to-leading order potentials [11]. The corresponding computations were

carried out in Refs. [12 14] and are completed [13, 14]. Using the conventions from [13] the resulting NLL order renormalization group equation (RGE) for the Wilson coefficient $c_{1}$ of 
the current $\mathbf{J}_{1, \mathbf{p}}$ has the form $\left(\mathbf{S}^{2}=2\right)$

$$
\left(\nu \frac{\partial}{\partial \nu} \ln \left[c_{1}(\nu)\right]\right)^{\mathrm{NLL}}=-\frac{\mathcal{V}_{c}^{(s)}(\nu)}{16 \pi^{2}}\left[\frac{\mathcal{V}_{c}^{(s)}(\nu)}{4}+\mathcal{V}_{2}^{(s)}(\nu)+\mathcal{V}_{r}^{(s)}(\nu)+\mathbf{S}^{2} \mathcal{V}_{s}^{(s)}(\nu)\right]+\frac{1}{2} \mathcal{V}_{k, \mathrm{eff}}^{(s)}(\nu)
$$

where $\nu$ is the vNRQCD velocity renormalization parameter that is conveniently used to parametrize the correlation between soft and ultrasoft dynamical scales within the renormalized effective theory [11]. The generalization of Eq. (2) for currents describing pairs of quarks and colored scalars in any angular momentum and spin state $\left({ }^{2 s+1} L_{J}\right)$ were derived in Ref. [15].

In Eq. (2) the term $\mathcal{V}_{c}^{(s)}$ is the Wilson coefficient of the Coulomb potential $\propto 1 / \mathbf{k}^{2}$, and $\mathcal{V}_{2}^{(s)}$ and $\mathcal{V}_{r}^{(s)}$ are the coefficients of the $\mathcal{O}\left(v^{2}\right)$ potentials with the momentum structure $1 / m^{2}$ and $\left(\mathbf{p}^{2}+\mathbf{p}^{\prime 2}\right) /\left(2 m^{2} \mathbf{k}^{2}\right)$, respectively, $m$ being the heavy quark mass. The term $\mathcal{V}_{s}^{(s)}$ is the coefficient of the spin-dependent potential that can contribute for spin triplet S-wave states. The coefficient $\mathcal{V}_{k, \text { eff }}^{(s)}$ combines Wilson coefficients from several non-Abelian $1 / m|\mathbf{k}|$ potentials at $\mathcal{O}(v)$. At LL order it has the form

$$
\mathcal{V}_{k, \text { eff }}^{(s)}(\nu)=\alpha_{s}(m \nu)^{2} \frac{C_{F}}{2}\left(C_{F}-2 C_{A}\right)+\left(\mathcal{V}_{k, \text { eff }}^{(s)}(\nu)\right)_{u s} .
$$

The first term arises from soft renormalization and the second represents the evolution from ultrasoft UV divergences. ${ }^{1}$ The terms in Eq. (3) are defined such that the ultrasoft terms have zero matching condition at $\nu=1,\left(\mathcal{V}_{k, \text { eff }}^{(s)}(1)\right)_{u s}=0$. The superscripts $(s)$ refer to the color singlet state of the quark pair.

At NNLL order there are two types of contributions that have to be considered for the evolution of $c_{1}$. The first arises from three-loop vertex diagrams that come from insertions of subleading soft matrix element corrections to the potentials and from insertions of potentials with additional exchange of ultrasoft gluons. The corresponding results were determined in Ref. [16] and are referred to as the non-mixing contributions as they affect the evolution of $c_{1}$ directly through UV-divergences. The second type of contributions arises from the subleading evolution of the potential Wilson coefficients that appear in the NLL order RGE shown in Eq. (2). They are referred to as the mixing contributions as they affect the evolution of $c_{1}$ indirectly. Except for the coefficient of the Coulomb potential $\mathcal{V}_{c}^{(s)}$ [13, 17] and for the spin-dependent potential $\mathcal{V}_{s}^{(s)}$ [18] no complete determination for the subleading evolution exists at present.

The analysis of the three-loop (non-mixing) terms in Ref. [16] showed that the contributions involving the exchange of ultrasoft gluons are more than an order of magnitude larger than those arising from soft matrix element insertions and in fact similar in size to the previously known NLL contributions. The reason is related to the larger size of the ultrasoft coupling $\alpha_{s}\left(m \nu^{2}\right)$ and to a rather large coefficient multiplying the ultrasoft contributions. These large ultrasoft contributions are responsible for an uncertainty in the normalization of the most up-to-date top pair threshold cross section prediction of at best $6 \%$ [19], which is quite far from the required precision (see also Ref. [10]). From this analysis it is reasonable to assume that the ultrasoft effects, which form a gauge-invariant subset, also dominate the

1 There is a misprint in Eq.(4) of Ref. 16] where a factor 1/2 is missing for the soft contributions. All numerical computations in Ref. [16] were carried out with the correct result. 
mixing contributions. This is also consistent with the small numerical effects [20] of the NLL evolution of the spin-dependent coefficient $\mathcal{V}_{s}^{(s)}$ [18], which is dominated by soft effects and receives ultrasoft contributions only indirectly through mixing.

In Ref. [21] we have computed the ultrasoft contribution to the NLL anomalous dimensions of the spin-independent $1 / m^{2}$ potential coefficients $\mathcal{V}_{2}^{(s)}$ and $\mathcal{V}_{r}^{(s)}$. Here UV-divergent diagrams with two ultrasoft loops contribute directly to their anomalous dimensions. These NLL ultrasoft corrections were indeed large and found to be similar in size to the previously known LL order evolution. Their overall contribution in the NNLL mixing corrections to the NLL RGE in Eq. (2), however, turned out to be quite small due to the $1 / 16 \pi^{2}$ suppression factor.

In this work we complete the determination of the NNLL ultrasoft corrections to the anomalous dimension of $c_{1}$ by the computation of the NLL ultrasoft renormalization group $(\mathrm{RG})$ evolution of the coefficients $\left(\mathcal{V}_{k, \text { eff }}^{(s)}\right)_{u s}$. Since its contribution in Eq. (2) is substantially larger than those of the $\mathcal{V}_{2, r}^{(s)}$, the impact of the NLL ultrasoft corrections can be expected to be sizeable and might compensate the large ultrasoft NNLL non-mixing corrections to $c_{1}$ determined in Ref. [16]. As we show in this work, this is indeed the case as the results of this work substantially stabilize the RG evolution of $c_{1}$. Our result will contribute to a reduction of the theoretical uncertainty of current RGI predictions for the heavy quark pair threshold production rate and in particular for top quark pair production at a future linear collider.

This work is organized as follows: In Sec. II we briefly review the effective theory setup used for our work and define the relevant operators in the vNRQCD Lagragian. The ultrasoft renormalization procedure with regard to the $1 / m|\mathbf{k}|$ potentials is explained in Sec. [II] Section IV gives a short overview of the respective calculation at the LL level in Feynman gauge, before we present the new calculation at NLL order in Sec. V]. In Sec. VI we summarize the results for the $1 / m|\mathbf{k}|$ and $1 / m^{2}$ potentials and present the complete ultrasoft NNLL mixing contribution to the running of the current coefficient $c_{1}$. Sec. VII contains a numerical analysis of our results and Sec. VII our conclusion.

\section{THEORETICAL SETUP}

The vNRQCD Lagrangian is organized as an expansion in the heavy quark velocity $v$. It consists of three mayor parts [11, 13, 22],

$$
\mathcal{L}_{\mathrm{vNRQCD}}=\mathcal{L}_{u}+\mathcal{L}_{p}+\mathcal{L}_{s}
$$

containing kinetic terms and ultrasoft interactions $\left(\mathcal{L}_{u}\right)$, potential interactions $\left(\mathcal{L}_{p}\right)$ and interactions involving soft degrees of freedom $\left(\mathcal{L}_{s}\right)$, respectively. The ultrasoft term $\mathcal{L}_{u}$ has the form

$$
\mathcal{L}_{u}=\sum_{\mathbf{p}}\left\{\psi_{\mathbf{p}}^{\dagger}\left[i D^{0}-\frac{(\mathbf{p}-i \mathbf{D})^{2}}{2 m}+\frac{\mathbf{p}^{4}}{8 m^{3}}+\ldots\right] \psi_{\mathbf{p}}+(\psi \rightarrow \chi, T \rightarrow \bar{T})\right\}-\frac{1}{4} G^{\mu \nu} G_{\mu \nu}+\ldots
$$

where $D^{\mu}=\partial^{\mu}+i g_{U} A^{\mu}(x)$ is the ultrasoft gauge-covariant derivative, $g_{U}$ is the ultrasoft coupling constant, and $G^{\mu \nu}$ is the ultrasoft field strength tensor. Besides the propagation of the heavy quarks $\mathcal{L}_{u}$ describes their interaction with ultrasoft gluons. 
The potential term $\mathcal{L}_{p}$ contains the potential interactions between the quark and the antiquark and can be written as

$$
\mathcal{L}_{p}=\mathcal{L}_{p V}+\mathcal{L}_{p u}+\mathcal{L}_{p k}
$$

where

$$
\mathcal{L}_{p V}=-\sum_{\mathbf{p}, \mathbf{p}^{\prime}} V_{\alpha \beta \lambda \tau}\left(\mathbf{p}, \mathbf{p}^{\prime}\right) \psi_{\mathbf{p}^{\prime} \alpha}^{\dagger} \psi_{\mathbf{p} \beta} \chi_{-\mathbf{p}^{\prime} \lambda}^{\dagger} \chi_{-\mathbf{p} \tau}+\ldots
$$

consists of potential four-quark operators. They depend on the soft three-momentum labels $\mathbf{p}$ and $\mathbf{p}^{\prime}$ of the heavy quark fields, and the coefficients have the form

$$
\begin{aligned}
V_{\alpha \beta \lambda \tau}\left(\mathbf{p}, \mathbf{p}^{\prime}\right)= & \left(T_{\alpha \beta}^{A} \otimes \bar{T}_{\lambda \tau}^{A}\right)\left[\frac{\mathcal{V}_{c}^{(T)}}{\mathbf{k}^{2}}+\frac{\mathcal{V}_{r}^{(T)}\left(\mathbf{p}^{2}+\mathbf{p}^{\prime 2}\right)}{2 m^{2} \mathbf{k}^{2}}+\frac{\mathcal{V}_{2}^{(T)}}{m^{2}}+\ldots\right] \\
& +\left(1_{\alpha \beta} \otimes \overline{1}_{\lambda \tau}\right)\left[\frac{\mathcal{V}_{c}^{(1)}}{\mathbf{k}^{2}}+\frac{\mathcal{V}_{r}^{(1)}\left(\mathbf{p}^{2}+\mathbf{p}^{\prime 2}\right)}{2 m^{2} \mathbf{k}^{2}}+\frac{\mathcal{V}_{2}^{(1)}}{m^{2}}+\ldots\right]
\end{aligned}
$$

the terms $\alpha, \beta, \lambda, \tau$ being color indices and $\mathbf{k} \equiv \mathbf{p}^{\prime}-\mathbf{p}$. The ellipses in Eq. (8) refer to spin-dependent $\mathcal{O}\left(v^{2}\right)$ potentials, that are not relevant in this work, as well as to higher orders in the $v$ expansion. The leading order terms in Eq. (8) in the velocity power counting are the Coulomb potential operators. Their LL coefficients are $\mathcal{V}_{c}^{(T)}(\nu)=4 \pi \alpha_{s}(m \nu)$ and $\mathcal{V}_{c}^{(1)}(\nu)=0$, where $\nu$ is the vNRQCD velocity renormalization scaling parameter [11].

The term $\mathcal{L}_{p u}$ includes the higher order terms in the multipole expansion related to the potentials in Eq. (8)). It is fixed by reparametrization and (ultrasoft) gauge invariance of $\mathcal{L}_{p}[22]:$

$$
\begin{aligned}
\mathcal{L}_{p u}= & \frac{2 i \mathcal{V}_{c}^{(T)} f^{A B C}}{\mathbf{k}^{4}} \mathbf{k} \cdot\left(g \mathbf{A}^{C}\right) \psi_{\mathbf{p}^{\prime}}^{\dagger} T^{A} \psi_{\mathbf{p}} \chi_{-\mathbf{p}^{\prime}}^{\dagger} \bar{T}^{B} \chi_{-\mathbf{p}}+ \\
& +\mathcal{V}_{c}^{(T)} \psi_{\mathbf{p}^{\prime}}^{\dagger}\left[\frac{i \mathbf{k} \cdot \overleftrightarrow{\nabla}}{\mathbf{k}^{4}}-\frac{\overleftrightarrow{\nabla}^{2}}{2 \mathbf{k}^{4}}+2 \frac{(\mathbf{k} \cdot \overleftrightarrow{\nabla})^{2}}{\mathbf{k}^{6}}\right] T^{A} \psi_{\mathbf{p}} \chi_{-\mathbf{p}^{\prime}}^{\dagger} \bar{T}^{A} \chi_{-\mathbf{p}}+ \\
& +\mathcal{V}_{c}^{(T)} \psi_{\mathbf{p}^{\prime}}^{\dagger} T^{A} \psi_{\mathbf{p}} \chi_{-\mathbf{p}^{\prime}}^{\dagger}\left[\frac{-i \mathbf{k} \cdot \overleftrightarrow{\nabla}}{\mathbf{k}^{4}}-\frac{\ddot{\nabla}^{2}}{2 \mathbf{k}^{4}}+2 \frac{(\mathbf{k} \cdot \overleftrightarrow{\nabla})^{2}}{\mathbf{k}^{6}}\right] \bar{T}^{A} \chi_{-\mathbf{p}}+\ldots
\end{aligned}
$$

The first term in Eq. (9) describes the coupling of an ultrasoft gluon to the Coulomb potential, the other terms are four-quark operators with ultrasoft derivatives $\vec{\nabla}=\vec{\nabla}+\overleftarrow{\nabla}$ acting on the fermion fields to the left and to the right. The associated Feynman rules can be found in App. A,

In contrast to early works [11, 12, 22], we omit potential terms of the form

$$
\left(T_{\alpha \beta}^{A} \otimes \bar{T}_{\lambda \tau}^{A}\right) \frac{\mathcal{V}_{k}^{(T)} \pi^{2}}{m|\mathbf{k}|}+\left(1_{\alpha \beta} \otimes \overline{1}_{\lambda \tau}\right) \frac{\mathcal{V}_{k}^{(1)} \pi^{2}}{m|\mathbf{k}|}
$$

in Eq. (8). In the term

$$
\mathcal{L}_{p k}=\sum_{i, X} \mathcal{V}_{k i}^{(X)} \mathcal{O}_{k i}^{(X)}+\ldots
$$


we instead introduce the sum operators $\mathcal{O}_{k i}$ as they are suitable to carry out the ultrasoft renormalization procedure [13, 16]. These sum operators generate a potential $V_{k} \propto 1 / m\left(\mathbf{k}^{2}\right)^{\frac{5-d}{2}}$ upon summation of the intermediate soft label momentum q, when inserted into vNRQCD matrix elements. The ellipses in Eq. (11) indicate other sum operators [13, 16] which we, however, do not need to consider in this work. For the sum operators needed in our work we use the following operator basis, $\mu_{S}=m \nu$ being the soft renormalization scale:

$$
\begin{aligned}
& \mathcal{O}_{k 1}^{(1)}=-\frac{\left[\mathcal{V}_{c}^{(T)}(\nu)\right]^{2} \mu_{S}^{4 \epsilon}}{m} 1 \otimes \overline{1} \sum_{\mathbf{p}, \mathbf{p}^{\prime}, \mathbf{q}}\left(f_{0}+f_{1}+2 f_{2}\right)\left[\psi_{\mathbf{p}^{\prime}}^{\dagger}, \psi_{\mathbf{p}} \chi_{-\mathbf{p}^{\prime}}^{\dagger} \chi_{-\mathbf{p}}\right], \\
& \mathcal{O}_{k 2}^{(T)}=-\frac{\left[\mathcal{V}_{c}^{(T)}(\nu)\right]^{2} \mu_{S}^{4 \epsilon}}{m} T^{A} \otimes \bar{T}^{A} \sum_{\mathbf{p}, \mathbf{p}^{\prime}, \mathbf{q}}\left(f_{1}+f_{2}\right)\left[\psi_{\mathbf{p}^{\prime}}^{\dagger} \psi_{\mathbf{p}} \chi_{-\mathbf{p}^{\prime}}^{\dagger} \chi_{-\mathbf{p}}\right], \\
& \mathcal{O}_{k 3}^{(1)}=-\frac{\left[\mathcal{V}_{c}^{(T)}(\nu)\right]^{2} \mu_{S}^{4 \epsilon}}{m} 1 \otimes \overline{1} \sum_{\mathbf{p}, \mathbf{p}^{\prime}, \mathbf{q}}\left(f_{0}+f_{1}\right)\left[\psi_{\mathbf{p}^{\prime}}^{\dagger} \psi_{\mathbf{p}} \chi_{-\mathbf{p}^{\prime}}^{\dagger} \chi_{-\mathbf{p}}\right] . \\
& \mathcal{O}_{k 3}^{(T)}=-\frac{\left[\mathcal{V}_{c}^{(T)}(\nu)\right]^{2} \mu_{S}^{4 \epsilon}}{m} T^{A} \otimes \bar{T}^{A} \sum_{\mathbf{p}, \mathbf{p}^{\prime}, \mathbf{q}}\left(f_{0}+f_{1}\right)\left[\psi_{\mathbf{p}^{\prime}}^{\dagger} \psi_{\mathbf{p}} \chi_{-\mathbf{p}^{\prime}}^{\dagger} \chi_{-\mathbf{p}}\right] .
\end{aligned}
$$

The functions $f_{i}$ depend on the external soft three-momentum labels $\mathbf{p}, \mathbf{p}^{\prime}$ and the intermediate soft three-momentum label q. They are defined as [13]

$$
\begin{aligned}
& f_{0}=\frac{\mathbf{p}^{\prime} \cdot(\mathbf{q}-\mathbf{p})}{(\mathbf{q}-\mathbf{p})^{4}\left(\mathbf{q}-\mathbf{p}^{\prime}\right)^{2}}+\left(\mathbf{p} \leftrightarrow \mathbf{p}^{\prime}\right), \\
& f_{1}=\frac{\mathbf{q} \cdot(\mathbf{q}-\mathbf{p})}{(\mathbf{q}-\mathbf{p})^{4}\left(\mathbf{q}-\mathbf{p}^{\prime}\right)^{2}}+\left(\mathbf{p} \leftrightarrow \mathbf{p}^{\prime}\right), \\
& f_{2}=\frac{\left(\mathbf{q}-\mathbf{p}^{\prime}\right) \cdot(\mathbf{q}-\mathbf{p})}{(\mathbf{q}-\mathbf{p})^{4}\left(\mathbf{q}-\mathbf{p}^{\prime}\right)^{4}}\left(\mathbf{q}^{2}-\frac{\mathbf{p}^{\prime 2}}{2}-\frac{\mathbf{p}^{2}}{2}\right) .
\end{aligned}
$$

In four-quark matrix elements the sum over $\mathbf{q}$ is understood to be replaced by a soft loop integral [11], i.e.

$$
\sum_{\mathbf{q}} \rightarrow \int \frac{d^{d-1} q}{(2 \pi)^{d-1}}
$$

The $\mathbf{q}$ integrals over the $f_{i}$ 's in $d-1$ spatial dimensions yield functions that only depend on $\mathrm{k}=\mathbf{p}^{\prime}-\mathbf{p}$,

$$
\begin{aligned}
\int \frac{d^{d-1} q}{(2 \pi)^{d-1}} f_{0} & =\frac{1}{2}|\mathbf{k}|^{d-5}[f(1,1)+f(1,2)] \stackrel{d \rightarrow 4}{\longrightarrow} \frac{1}{16|\mathbf{k}|} \\
\int \frac{d^{d-1} q}{(2 \pi)^{d-1}} f_{1} & =\frac{1}{2}|\mathbf{k}|^{d-5}[3 f(1,1)-f(1,2)] \stackrel{d \rightarrow 4}{\longrightarrow} \frac{3}{16|\mathbf{k}|}, \\
\int \frac{d^{d-1} q}{(2 \pi)^{d-1}} f_{2} & =\frac{1}{4}|\mathbf{k}|^{d-5}[2 f(1,1)-4 f(1,2)+f(2,2)] \stackrel{d \rightarrow 4}{\longrightarrow} \frac{1}{16|\mathbf{k}|},
\end{aligned}
$$

where

$$
f(a, b)=\frac{\Gamma\left(a+b-\frac{d-1}{2}\right) \Gamma\left(\frac{d-1}{2}-a\right) \Gamma\left(\frac{d-1}{2}-b\right)}{\Gamma(a) \Gamma(b) \Gamma(d-1-a-b)(4 \pi)^{\frac{d-1}{2}}}
$$


They reproduce for $d \rightarrow 4$ the $1 /|\mathbf{k}|$ potentials mentioned in Eq. (10). For simplicity we call the sum operators $\mathcal{O}_{k, i}^{(X)}$ also $V_{k}$ potentials in the rest of this work. The operators $\mathcal{O}_{k 1}^{(1)}$ and $\mathcal{O}_{k 2}^{(T)}$ have been introduced before in Ref. [13] for the LL ultrasoft renormalization. The operators $\mathcal{O}_{k 3}^{(1, T)}$ are new and required for the presentation of our computations at NLL order.

Unlike the corresponding operators in Eq. (10) the sum operators can be renormalized consistently beyond one-loop level [13, 23. . By including the renormalized factor $\left[\mathcal{V}_{c}^{(T)}(\nu)\right]^{2}$ in the definition of the $\mathcal{O}_{k i}$ we anticipate a factorization of the soft (LL) contributions (encoded in $\mathcal{V}_{c}^{(T)}(\nu)$ ) and the ultrasoft contributions (encoded in $\mathcal{V}_{k i}^{(X)}(\nu)$ ) to their RG running. An analogous factorization occurs for the $1 / m^{2}$ potentials $\mathcal{V}_{r}$ and $\mathcal{V}_{2}$ [13, 21]. ${ }^{2}$ In this paper we compute the (ultrasoft) NLL running of the $\mathcal{V}_{k i}(\nu)$. The LL running was determined in Ref. [13] in Coulomb gauge.

Using the equation

$$
\left[\begin{array}{c}
V_{\text {singlet }} \\
V_{\text {octet }}
\end{array}\right]=\left[\begin{array}{cc}
1 & -C_{F} \\
1 & \frac{1}{2} C_{A}-C_{F}
\end{array}\right]\left[\begin{array}{c}
V_{1 \otimes 1} \\
V_{T \otimes T}
\end{array}\right]
$$

for vectors in color space, we can easily convert the potential operators in Eqs. (8), (91), (12) from the basis formed by the two $3 \otimes \overline{\mathbf{3}}$ color structures $1 \otimes \overline{1}$ and $T^{A} \otimes \bar{T}^{A}$ into the color singlet/octet basis used for physical applications. In this paper only the gauge invariant color singlet configuration of the potentials (and the $\mathcal{O}_{k i}$ ) will be considered. In order to provide compact results we will therefore restrict ourselves to the renormalization of the singlet operators

$$
\begin{aligned}
& \mathcal{O}_{k 1}^{(s)}=-\frac{\left[\mathcal{V}_{c}^{(T)}(\nu)\right]^{2} \mu_{S}^{4 \epsilon}}{m} \mathrm{P}_{\text {Singlet }} \sum_{\mathbf{p}, \mathbf{p}^{\prime}, \mathbf{q}}\left(f_{0}+f_{1}+2 f_{2}\right)\left[\psi_{\mathbf{p}^{\prime}}^{\dagger} \psi_{\mathbf{p}} \chi_{-\mathbf{p}^{\prime}}^{\dagger} \chi_{-\mathbf{p}}\right], \\
& \mathcal{O}_{k 2}^{(s)}=-\frac{\left[\mathcal{V}_{c}^{(T)}(\nu)\right]^{2} \mu_{S}^{4 \epsilon}}{m} \mathrm{P}_{\text {Singlet }} \sum_{\mathbf{p}, \mathbf{p}^{\prime}, \mathbf{q}}\left(f_{1}+f_{2}\right)\left[\psi_{\mathbf{p}^{\prime}}^{\dagger} \psi_{\mathbf{p}} \chi_{-\mathbf{p}^{\prime}}^{\dagger} \chi_{-\mathbf{p}}\right], \\
& \mathcal{O}_{k 3}^{(s)}=-\frac{\left[\mathcal{V}_{c}^{(T)}(\nu)\right]^{2} \mu_{S}^{4 \epsilon}}{m} \mathrm{P}_{\text {Singlet }} \sum_{\mathbf{p}, \mathbf{p}^{\prime}, \mathbf{q}}\left(f_{0}+f_{1}\right)\left[\psi_{\mathbf{p}^{\prime}}^{\dagger} \psi_{\mathbf{p}} \chi_{-\mathbf{p}^{\prime}}^{\dagger} \chi_{-\mathbf{p}}\right] .
\end{aligned}
$$

Here $\mathrm{P}_{\text {Singlet }}=\left(1-2 \frac{C_{F}}{C_{A}}\right) 1 \otimes \overline{1}-\frac{2}{C_{A}} T^{A} \otimes \bar{T}^{A}$ is the color singlet projection operator.

All fields, couplings and Wilson coefficients in the above Lagrangian are to be understood as bare quantities unless stated otherwise. For the renormalized quantities, indicated here

2 A complete account on how this factorization arises requires the introduction of additional sum operators with four heavy quark and two soft fields, which, analogous to the $\mathcal{O}_{k i}$, absorb the UV divergences of vNRQCD diagrams with external soft fields and ultrasoft and potential loops. By forming a soft tadpole these operators contribute soft mixing terms to the anomalous dimension of the $\mathcal{O}_{k i}$. Together with the contributions in this work they produce the factorized running of the coefficient $\left[\mathcal{V}_{c}^{(T)}(\nu)\right]^{2} \mathcal{V}_{k i}^{(X)}(\nu)$. This approach has been adopted in Ref. 21]. We do not follow this approach here and refer to Ref. [23] for a detailed discussion. In the two-stage matching approach adopted in "potential" NRQCD (pNRQCD) 24], this kind of factorization in the potential coefficients is implemented by construction. 
by the index $R$, we chose the usual conventions in $d=4-2 \epsilon$ dimensions:

$$
\begin{aligned}
& g_{U}=\mu_{U}^{\epsilon} Z_{g} g_{U}^{R}, \quad g_{S}=\mu_{S}^{\epsilon} Z_{g} g_{S}^{R}, \\
& \psi_{\mathbf{p}}=Z_{\psi, \mathbf{p}}^{1 / 2} \psi_{\mathbf{p}}^{R}, \quad Z_{\psi, \mathbf{p}}=1+\delta Z_{\psi, \mathbf{p}}, \quad(\psi \rightarrow \chi), \\
& A^{\mu}=Z_{A}^{1 / 2} A_{R}^{\mu}, \quad Z_{A}=1+\delta Z_{A} \text {, } \\
& \mathcal{V}_{i}=\mu_{S}^{2 \epsilon}\left(\mathcal{V}_{i, R}+\delta \mathcal{V}_{i}\right), \quad \mathcal{V}_{k i}=\mu_{S}^{2 \epsilon}\left(\mathcal{V}_{k i, R}+\delta \mathcal{V}_{k i}\right) \text {, }
\end{aligned}
$$

where $\mu_{S}=m \nu$ and $\mu_{U}=m \nu^{2}$ are the soft and ultrasoft renormalization scales, respectively. For convenience, we will drop the index $R$ throughout this paper and only deal with $\overline{\mathrm{MS}}$ renormalized quantities in the following. We will moreover use the notation

$$
\alpha_{S} \equiv \alpha_{s}(m \nu)=\frac{g_{S}^{2}}{4 \pi}, \quad \alpha_{U} \equiv \alpha_{s}\left(m \nu^{2}\right)=\frac{g_{U}^{2}}{4 \pi} .
$$

\section{RENORMALIZATION PROCEDURE}

The aim of this work is to determine the ultrasoft anomalous dimension of the sum operator coefficients $\mathcal{V}_{k i}^{(1, T)}(\nu)$ at NLL order. We work in Feynman gauge and use the $\overline{\mathrm{MS}}$ renormalization scheme in combination with dimensional regularization $(d=4-2 \epsilon)$. All operators $\mathcal{O}_{k i}^{(1, T)}$ in Eq. (18) have zero matching condition at the hard scale, i.e. $\mathcal{V}_{k i}^{(1, T)}(\nu=1)=0$ [13].

Purely ultrasoft loop corrections to potential operators in vNRQCD are inevitably suppressed by two powers of the velocity $v .{ }^{3}$ We therefore need at least one additional compensating factor $\sim \alpha_{S} / v$ from a potential loop in order to obtain the correct $v$-scaling of a $V_{k}$ potential [11, 22]. This is why the $V_{k}$ potential first receives a running at $\mathcal{O}\left(\alpha_{S}^{2}\right)$ as indicated by the factor $\left(\mathcal{V}_{c}^{(T)}\right)^{2}$ in the definition of the $\mathcal{O}_{k i}^{(1, T)}$.

Figure 3 and table $\llbracket$ show relevant UV-divergent $\mathcal{O}\left(\alpha_{S}^{2} \alpha_{U}\right)$ and $\mathcal{O}\left(\alpha_{S}^{2} \alpha_{U}^{2}\right)$ diagrams, which contribute in Feynman gauge to the anomalous dimension of the $V_{k}$ potentials at ultrasoft LL and NLL level, respectively. Each diagram consists of one UV-finite potential loop and UV-divergent ultrasoft gluon attachments. Many diagrams also contain ultrasoft derivative operators from higher orders in the multipole expansion. They are depicted as nabla $(\nabla)$ and Laplace $(\Delta)$ operator symbols on heavy quark lines or on potential vertices. The operator insertions on the lines represent relativistic corrections to the heavy quark kinetic term $\propto\left(\partial_{0}-\frac{\mathbf{p}^{2}}{2 m}\right)$ (see Eq. (5) ) due to ultrasoft momentum components flowing through the heavy quark propagators. The potentials with ultrasoft derivatives are defined in Eq. (9) and represent corrections due to ultrasoft momentum exchange between quark and antiquark. The corresponding Feynman rules are given in App. $\mathrm{A}$. ${ }^{4}$ In Fig. 1 we show our generic choice for the external energy and momenta running through the diagrams with a potential loop. Note that vNRQCD as reviewed in Sec. II is formulated in the center of mass frame. The little arrows on the heavy quark lines denote positive energy flow and are suppressed in the other graphs shown in this paper.

$3 \operatorname{At} \mathcal{O}\left(v^{0}\right)$ the interaction between ultrasoft $A^{0}$ gluons and heavy quarks can be removed by a field redefinition that leaves physical predictions unchanged [11, 23].

4 Such derivative operators also exist for heavy quark lines/vertices which do not carry ultrasoft momentum components, but then they evaluate to zero. This needs to be taken into account when showing the equivalence of diagrams that differ by the routing of ultrasoft momenta. 


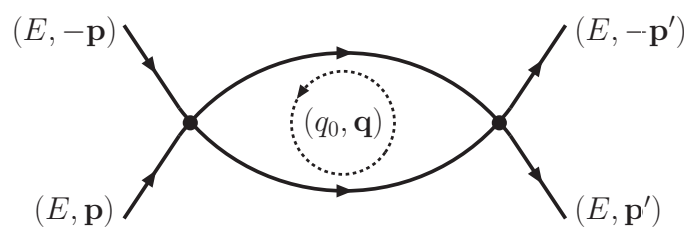

FIG. 1: External energy/momenta convention for all diagrams with potential loop.

As demonstrated in Ref. [13] the additional potential loop entails the renormalization of the $\mathcal{O}_{k i}^{(1, T)}$ sum operators instead of the potentials in Eq. (10). The reason becomes apparent at the three-loop level, when the potential loop appears together with two ultrasoft loops. Some typical example diagrams are shown in table 【. After the integrations over ultrasoft and potential loop momenta their leading divergences typically take the schematic form [13]

$$
\frac{1}{|\mathbf{k}|}\left(\frac{\mu_{S}^{2}}{\mathbf{k}^{2}}\right)^{\epsilon}\left[\frac{1}{2 \epsilon^{2}}\left(\frac{\mu_{U}^{2}}{E^{2}}\right)^{2 \epsilon}-\frac{1}{\epsilon^{2}}\left(\frac{\mu_{U}^{2}}{E^{2}}\right)^{\epsilon}\right]=\frac{1}{|\mathbf{k}|}\left[-\frac{1}{2 \epsilon^{2}}-\frac{1}{2 \epsilon} \ln \left(\frac{\mu_{S}^{2}}{\mathbf{k}^{2}}\right)+\ldots\right],
$$

where $E$ represents the ultrasoft scale $\sim m v^{2}$, i.e. the kinetic energy of the external heavy quarks. Inside the square brackets the first term arises from the three-loop diagrams and the second from two-loop diagrams with one-loop counterterms to remove subdivergences. The factor in front of the square brackets on the left-hand-side comes from the finite potential loop. The $\ln \left(\mu_{S}^{2} / \mathbf{k}^{2}\right)$-dependent $1 / \epsilon$-pole on the right-hand-side of Eq. (21) cannot be absorbed into counterterms for potentials of the type in Eq. (10), because this would cause inconsistent anomalous dimensions. This is the reason, why the sum operators $\mathcal{O}_{k i}$ are essential for a consistent renormalization beyond the one-loop level in vNRQCD. Upon summing the intermediate soft momentum label $\mathbf{q}$, they contain the proper potential prefactors in Eq. (21) in their operator structure. Therefore the $\delta \mathcal{V}_{k i}$ counterterms only absorb proper, scale independent UV poles and lead to consistent anomalous dimensions.

In practice we have to determine the $\delta \mathcal{V}_{k i}$ from the ultrasoft UV divergences of two- (LL) and three-loop (NLL) Feynman diagrams after the integration over the zero-component of the potential loop momentum $\left(q_{0}\right)$, which can be carried out using residues. The potential three-momentum (q) integration, however, is not carried out in the renormalization procedure as the q-dependence of the remaining UV-divergences has to be matched onto the label structures of the sum operators $\mathcal{O}_{k i}$. There is a technical subtlety concerning the vNRQCD multipole expansion related to taking residues with respect to the potential loop momentum $q_{0}$ in the upper or the lower complex half plane. Here differences can arise in the soft momentum label structure in terms of $\mathbf{p}, \mathbf{p}^{\prime}$ and $\mathbf{q}$ resulting from insertions of the ultrasoft derivative operators which are non-zero only when the corresponding quark line carries an ultrasoft momentum component. Upon carrying out the label sum the results are unique. To avoid redundancies in the operator structure of the $\mathcal{O}_{k i}$ we need to define a consistent prescription for the respective Feynman graphs in order to ensure, that the operator basis of the $\mathcal{O}_{k i}$ required for the renormalization is unique. We evaluate each Feynman diagram with a potential loop considered in this paper as follows $\left(q^{\mu}=\left(q_{0}, \mathbf{q}\right)\right.$ being the potential loop momentum):

1. Neglect the kinetic and potential multipole correction operator insertions for the moment. Pick one internal heavy quark or antiquark line (which carries momentum q) and choose an ultrasoft momentum routing such that this heavy quark/antiquark line 
does not carry any ultrasoft momentum. Now, according to the chosen routing insert the required ultrasoft derivative operators in all possible ways.

2. Compute the residue of the pole in the complex $q_{0}$ plane associated with the propagator without ultrasoft momentum picked in step 1 for the chosen routing and each vertex/insertion configuration. Multiply with the proper factor $( \pm 2 \pi i)$ according to the residue theorem depending on whether the pole lies in the upper or lower complex half plane.

3. Perform the ultrasoft loop integrals.

4. Repeat steps 1-3 for all internal heavy quark/antiquark lines. Sum all expressions obtained in this way and divide the result by two.

The factor $1 / 2$ from point 4 compensates for the overcounting, which arises in points 1 and 2 from closing the $q_{0}$ integration contour in the upper as well as in the lower complex half plane.

From a technical point of view this procedure corresponds to the renormalization of properly defined six-quark operators in order to absorb the ultrasoft UV divergences of the six-leg diagrams, which are generated from cutting the heavy quark/antiquark lines of the potential loop where the residues of step 2 are taken. This is the origin of the notation used in Fig. 2. The two heavy quark fields associated with the cut can then be contracted to form tadpole diagrams with four external legs, which is equivalent to carrying out the label sum for the sum operators. This procedure yields the same results as using the matrix elements of the sum-operators (see Ref. [23] for details). In Fig. 2 we visualize the terms arising in point 2 of the above caculational prescription using their analogy to the described six-leg diagrams.

\section{LL CALCULATION}

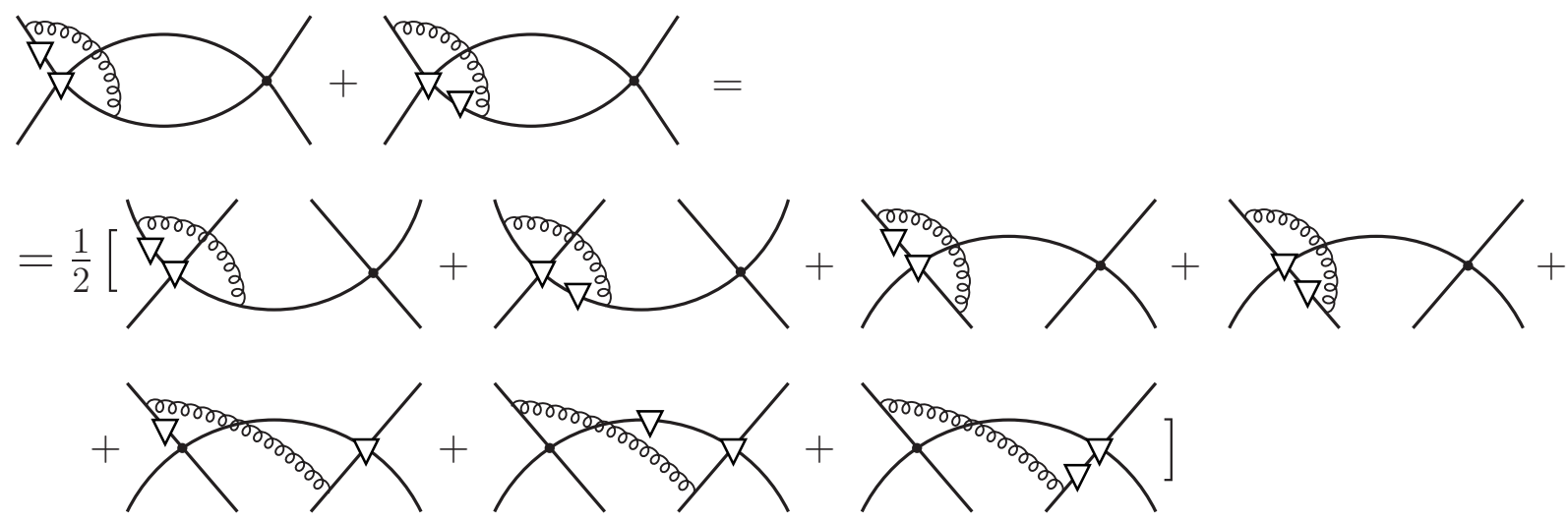

FIG. 2: Example for a calculation with one ultrasoft loop involving ultrasoft derivative operators. Each of the cut heavy quark/antiquark lines on the right-hand side corresponds to a $q_{0}$ residue to be taken at this line within the original closed potential loop diagram on the left-hand side according to step 2 of our calculational prescription. The color structure of the diagrams is understood to be not affected by the cutting, i.e. it is the same on both sides of the equation. 
In order to illustrate our computational procedure let us consider the simple case of the two $\mathcal{O}\left(\alpha_{S}^{2} \alpha_{U}\right)$ diagrams shown in Fig. 2. In Feynman gauge they contribute to the LL anomalous dimension of the $\mathcal{O}_{k i}$ and contain ultrasoft derivative operators. The outcome of steps 1 to 4 are sketched graphically in Fig. 2. Each six-leg diagram corresponds to picking up the residue of the cut heavy quark/antiquark line as described in step 2. The overall factor $1 / 2$ is due to step 4 . Including in addition all possible mirror-graphs and the analogous diagrams with one $\Delta$-operator, we obtain (up to an overall color factor not shown) the result $\frac{-i \mathcal{L}_{\alpha_{U}}^{2} \alpha_{U}}{\pi m \epsilon} \int \frac{d^{d-1} q}{(2 \pi)^{d-1}} \frac{2 f_{1}}{3}+\mathcal{O}\left(\epsilon^{0}\right)$, where in the integrand the term $\left(f_{0}+f_{1}\right) / 4$ arises from the residue in the upper complex half plane and $\left(-3 f_{0}+5 f_{1}\right) / 12$ from the residues in the the lower complex half plane. Upon integration over $\mathbf{q}$ both residue contributions agree.

We now briefly discuss the full LL ultrasoft renormalization of the $\mathcal{O}_{k i}$ in Feynman gauge; for details see Ref. [23]. This calculation is also interesting by itself since the available vNRQCD computations of Refs. [13, 22] were carried out in Coulomb gauge. Fig. [3] shows the relevant diagram topologies. Possible insertions of ultrasoft derivative operators are not shown.

a)

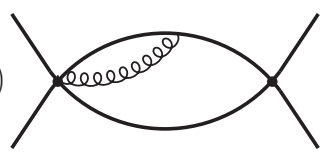

d)

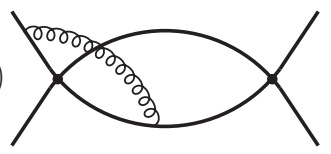

g)
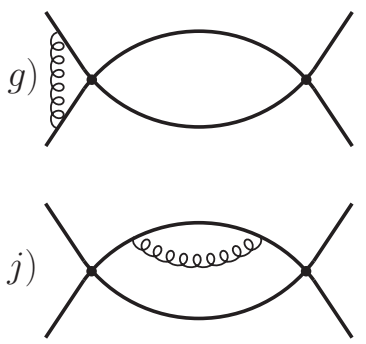

b)

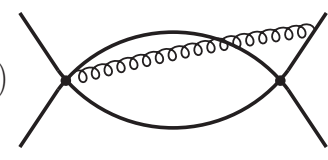

e)

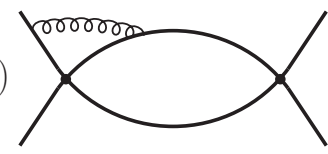

$h$
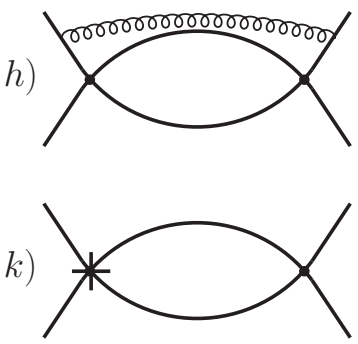

c)

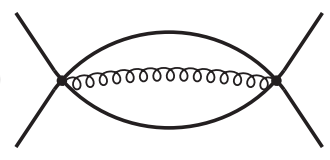

f)

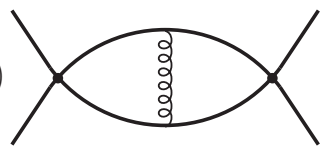

i)

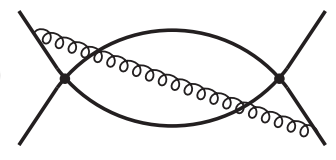

l)

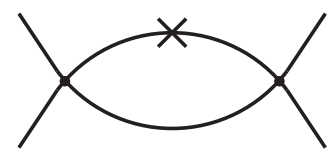

FIG. 3: Diagram topologies that generate the RG running of the $\mathcal{O}_{k i}$ at LL level. Up-down and left-right mirror graphs are understood.

The ultrasoft gluons in diagrams 3 a-c are spatial (A type) because there are no four-quark operators with one additional $A^{0}$ ultrasoft gluon in vNRQCD. Thus the vertices in these graphs already contribute two powers of $v$ to the velocity counting and we do not need to insert further derivative operators. On the other hand, diagrams with a spatial A gluon and topologies $\mathrm{d}-\mathrm{j}$ are either UV finite or their divergence is exactly canceled by one loop diagrams with $\delta \mathcal{V}_{r}, \delta \mathcal{V}_{2}$ or wavefunction counterterm insertions. The latter are depicted in diagrams $3 \mathrm{k}$ and 1 , respectively. The very same applies to the diagrams with an $A^{0}$ gluon for topologies $\mathrm{d}$-j, where two kinetic $\nabla$-operators are inserted on the heavy quark lines within the ultrasoft loop.

The remaining diagrams d-j with an $A^{0}$ gluon having the correct $v$ scaling contain either one $\Delta$ - or two $\nabla$-operator insertions, of which at least one is the $\nabla$-potential in Eq. (A5). 
Adding them to the $\mathbf{A}$ diagrams 3 a-c gives

$$
\begin{aligned}
& -i C_{A} \frac{\alpha_{U}\left(\mathcal{V}_{c}^{(T)}\right)^{2} \mu_{S}^{4 \epsilon}}{3 m \pi \epsilon} \\
& \quad \times \int \frac{d^{d-1} q}{(2 \pi)^{d-1}}\left[C_{F}\left(C_{A}-2 C_{F}\right)\left(f_{0}+f_{1}+2 f_{2}\right) 1 \otimes \overline{1}+\left(C_{A}-4 C_{F}\right)\left(f_{1}+f_{2}\right) T^{A} \otimes \bar{T}^{A}\right] .
\end{aligned}
$$

The result is exactly the same as obtained in Refs. [13, 22] where Coulomb gauge has been used. We note that the Coulomb gauge computation at LL order is substantially simpler since in Coulomb gauge $A^{0}$ gluons cannot propagate and the number of diagrams that have to be considered is reduced considerably. However, the Coulomb gauge calculation becomes a nightmare at the two-loop level and seems entirely unfeasible. We also note that we regularize IR divergences by adopting an off-shell configuration for the external heavy quark lines with $E \neq \mathbf{p}^{2} / 2 m=\left(\mathbf{p}^{\prime}\right)^{2} / 2 m$.

From Eq. (22) we can easily read off the already known $\mathcal{O}\left(\alpha_{U}\right)$ color singlet counterterms

$$
\delta \mathcal{V}_{k 1}^{(s)}=-C_{A} C_{F}\left(C_{A}-2 C_{F}\right) \frac{\alpha_{U}}{3 \pi \epsilon} ; \quad \delta \mathcal{V}_{k 2}^{(s)}=C_{A} C_{F}\left(C_{A}-4 C_{F}\right) \frac{\alpha_{U}}{3 \pi \epsilon} .
$$

\section{NLL CALCULATION}

Let us now consider the divergent $\mathcal{O}\left(\alpha_{S}^{2} \alpha_{U}^{2}\right)$ diagrams contributing to the ultrasoft NLL anomalous dimension of the $\mathcal{V}_{k i}$, i.e. diagrams with one potential and two ultrasoft loops. In table \ we have classified the relevant diagrams without gluon selfenergy with respect to their vertices and kinematic operator insertions. For each class one sample diagram with a specific configuration of vertices and insertions is shown graphically. Diagrams with all possible permutations of the vertices and different attachments of the gluons to the heavy quark lines are understood to belong to each class. The respective contribution in the last column of table \denotes the sum of the divergent parts of all class members. Contribution I covers classes 1 and 2, contribution iv covers classes 12 and 13. In the following we will call the diagram classes 1-8 "Abelian" and 9-14 "non-Abelian". The relevant Feynman gauge Feynman rules are found in Appendix A.

Besides the Abelian and non-Abelian diagrams in table \ there are $\mathcal{O}\left(\alpha_{S}^{2} \alpha_{U}^{2}\right)$ diagrams containing one-loop gluon selfenergy subdiagrams with ultrasoft light fermion, ghost and gluon bubbles. For the calculation of these diagrams we take the graphs with only one ultrasoft loop in Fig. 3 and replace the ultrasoft gluon propagators by their one-loop correction. Note that in Feynman gauge the selfenergy insertion is a non-diagonal matrix in the components of the ultrasoft gluon field and can couple the zero-component $\left(A^{0}\right)$ to the spatial components (A) of the ultrasoft gluon field due to its relativistic nature. Therefore we have to take into account diagrams with one $A^{0}$-vertex, one $\mathbf{A}$-vertex and a single $\nabla$ operator insertion, in addition to the diagrams we obtain by simply inserting the selfenergy bubble into the $\mathcal{O}\left(\alpha_{S}^{2} \alpha_{U}\right)$ diagrams of the previous section. After subtraction of the selfenergy subdivergences by adequate counterdiagrams the sum of all ultrasoft UV divergences 
from the three-loop $\mathcal{O}\left(\alpha_{S}^{2} \alpha_{U}^{2}\right)$ diagrams with a gluon selfenergy, reads:

$$
\begin{aligned}
& {\left[\frac{C_{A}\left(5 C_{A}-4 T n_{f}\right)}{72 \epsilon^{2}}-\frac{C_{A}\left(31 C_{A}-20 T n_{f}\right)}{216 \epsilon}\right] \frac{i \alpha_{U}^{2}\left(\mathcal{V}_{c}^{(T)}\right)^{2} \mu_{S}^{4 \epsilon}}{m \pi^{2}}} \\
& \quad \times \int \frac{d^{d-1} q}{(2 \pi)^{d-1}}\left[C_{F}\left(C_{A}-2 C_{F}\right)\left(f_{0}+f_{1}+2 f_{2}\right) 1 \otimes \overline{1}+\left(C_{A}-4 C_{F}\right)\left(f_{1}+f_{2}\right) T^{A} \otimes \bar{T}^{A}\right],
\end{aligned}
$$

where $T=\frac{1}{2}$ in the fundamental representation of $S U(3)$ and $n_{f}$ is the number of light quark flavors. The result contributes to the counterterms $\delta \mathcal{V}_{k 1}^{(1)}$ and $\delta \mathcal{V}_{k 2}^{(T)}$. The gauge invariant contributions from light fermion loops $\propto n_{f}$ in Eq. (24) were also determined in Coulomb gauge [25].

In table II we present results for the UV divergent parts of the Abelian and non-Abelian diagrams classified in table \after all one- and two-loop subdivergences that contribute to the renormalization of the $1 / \mathrm{m}^{2}$ potentials [12, 21] have been subtracted. ${ }^{5}$ The net contributions from the Abelian diagram classes 3 and 4 vanish, because the diagrams are either finite or the UV-divergences are cancelled exactly by the respective diagrams with $1 / \mathrm{m}^{2}$ potential counterterms. The results for I and II $+\mathrm{III}+\mathrm{IV}+\mathrm{V}$ also contain contributions from external wavefunction diagrams, i.e. heavy quark selfenergies on the four external legs. Two-loop heavy quark selfenergies with a triple gluon vertex vanish.

In order to avoid singularities of infrared origin we again assigned a uniform off-shellness to each of the four external legs. After the subtraction of subdivergences the logarithmic dependence on the off-shellness vanishes.

Note that apart from the external off-shellness the ultrasoft loops in the six-leg diagrams also involve the physical off-shellness of the intermediate (uncut) heavy quark line, 2(E$\frac{\mathbf{q}^{2}}{2 m}$ ). So the nontrivial ultrasoft two-loop diagrams that have to be calculated represent complicated multi-scale integrals.

We solved the integrals using partial fraction decomposition and integration by parts techniques. To handle the larger number of diagrams (corresponding to $\mathcal{O}\left(10^{4}\right)$ six-leg diagrams) we developed Mathematica codes for the generation and the evaluation of the corresponding amplitudes. For the $\epsilon=\frac{4-D}{2}$ expansion of the hypergeometric functions resulting from the two-loop integrations we used the Mathematica package HypExp 1.1 [26] and checked the results numerically. In order to automatize the determination of the color structures of the different three-loop topologies we moreover employed a few routines from the FeynCalc 4.1 package [27].

From the $\mathcal{O}\left(\alpha_{U}^{2}\right)$ UV divergences in table [1] and Eq. (24) we can now determine the

\footnotetext{
${ }^{5}$ The results in table $\llbracket$ can also be found in Eqs. (6.33) and (6.34) (Abelian contributions without wavefunction renormalization contributions) and table 6.9 (non-Abelian contributions) of Ref. 23]. In table 6.8 of Ref. 23] a result for the non-Abelian contributions is shown that is based on a calculation of six-leg diagrams, as explained at the end of Sec. III, using an off-shell configuration for the two lines coming from cutting the intermediate heavy quark propagator. This off-shell configuration disagrees with the on-shell condition arising from doing the potential energy integration by the residue theorem, and the result is not relevant for the computation intended in this work.
} 


\begin{tabular}{|c|c|c|c|c|c|c|}
\hline \multirow{2}{*}{ class } & \multirow{2}{*}{ example diagram } & \multicolumn{4}{|c|}{ vertices } & \multirow{2}{*}{ contrib. } \\
\hline & & potential & quark-gluon & 3 gluon & kin. ins. & \\
\hline 1 & & $\begin{array}{c}1 \times V_{c} \\
1 \times \mathbf{A} \cdot \mathbf{k}\end{array}$ & $\begin{array}{c}1 \times \mathbf{A} \\
2 \times A^{0}\end{array}$ & - & - & \multirow{2}{*}{$\mathrm{I}$} \\
\hline 2 & & $2 \times \mathbf{A} \cdot \mathbf{k}$ & $2 \times A^{0}$ & - & - & \\
\hline 3 & & $2 \times V_{c}$ & $\begin{array}{l}2 \times \mathbf{A} \\
2 \times A^{0} \\
\end{array}$ & - & - & 0 \\
\hline 4 & & $2 \times V_{c}$ & $4 \times A^{0}$ & - & $2 \times \nabla \cdot \mathbf{p}$ & 0 \\
\hline 5 & & $2 \times V_{c}$ & $4 \times A^{0}$ & - & $1 \times \nabla^{2}$ & II \\
\hline 6 & & $2 \times \nabla \cdot \mathbf{k}$ & $4 \times A^{0}$ & - & - & III \\
\hline 7 & & $1 \times V_{c}$ & $4 \times A^{0}$ & - & $1 \times \nabla \cdot \mathbf{p}$ & IV \\
\hline 8 & & $1 \times \nabla^{2}$ & $4 \times A^{0}$ & - & - & $\mathrm{V}$ \\
\hline 9 & & $2 \times V_{c}$ & $\begin{array}{l}2 \times \mathbf{A} \\
1 \times A^{0}\end{array}$ & $\mathbf{A}^{2} A^{0}$ & - & $\mathrm{i}$ \\
\hline 10 & & $2 \times V_{c}$ & $\begin{array}{l}1 \times \mathbf{A} \\
2 \times A^{0}\end{array}$ & $\mathbf{A}\left(A^{0}\right)^{2}$ & $1 \times \nabla \cdot \mathbf{p}$ & ii \\
\hline 11 & & $\begin{array}{c}1 \times V_{c} \\
1 \times \nabla \cdot \mathbf{k}\end{array}$ & $\begin{array}{l}1 \times \mathbf{A} \\
2 \times A^{0}\end{array}$ & $\mathbf{A}\left(A^{0}\right)^{2}$ & - & iii \\
\hline 12 & & $\begin{array}{c}1 \times V_{c} \\
1 \times \mathbf{A} \cdot \mathbf{k}\end{array}$ & $\begin{array}{l}1 \times \mathbf{A} \\
1 \times A^{0}\end{array}$ & $\mathbf{A}^{2} A^{0}$ & - & $i_{i}$ \\
\hline 13 & & $2 \times \mathbf{A} \cdot \mathbf{k}$ & $1 \times A^{0}$ & $\mathbf{A}^{2} A^{0}$ & - & \\
\hline 14 & & $1 \times V_{c}$ & $2 \times A^{0}$ & $\mathbf{A}\left(A^{0}\right)^{2}$ & $1 \times \nabla \cdot \mathbf{p}$ & $\mathrm{V}$ \\
\hline 15 & & $1 \times \mathbf{A} \cdot \mathbf{k}$ & $2 \times A^{0}$ & $\mathbf{A}\left(A^{0}\right)^{2}$ & - & vi \\
\hline
\end{tabular}

TABLE I: Diagram classes with one potential and two ultrasoft loops (without gluon self energy). Diagrams with all possible permutations of the vertices and different attachments of the gluons to the heavy quark lines are understood to belong to each class. The vNRQCD Feynman rules for the respective vertices, propagators and kinematic operator insertions are given in App. $\mathrm{A}$. 


\begin{tabular}{|c|c|}
\hline contribution & result $\cdot\left(\frac{i \mathcal{V}_{c}^{2} \alpha_{U}^{2}}{m}\right)^{-1}$ \\
\hline I & $\left(\frac{1}{8 \epsilon^{2}}-\frac{1}{4 \epsilon}\right) \frac{C_{A}^{2}}{\pi^{2}}\left[C_{F}\left(C_{A}-2 C_{F}\right)\left(f_{0}+f_{1}+2 f_{2}\right) 1 \otimes \overline{1}+\left(C_{A}-4 C_{F}\right)\left(f_{1}+f_{2}\right) T^{A} \otimes \bar{T}^{A}\right]$ \\
\hline $\mathrm{II}+\mathrm{III}+\mathrm{IV}+\mathrm{V}$ & $\left(-\frac{1}{24 \epsilon^{2}}+\frac{1}{8 \epsilon}\right) \frac{C_{A}^{2}}{\pi^{2}}\left[C_{F}\left(C_{A}-2 C_{F}\right)\left(f_{0}+f_{1}+2 f_{2}\right) 1 \otimes \overline{1}+\left(C_{A}-4 C_{F}\right)\left(f_{1}+f_{2}\right) T^{A} \otimes \bar{T}^{A}\right]$ \\
\hline $\mathrm{i}+\mathrm{ii}$ & $\frac{\mathbf{q}^{2}}{48 \epsilon(\mathbf{q}-\mathbf{p})^{2}\left(\mathbf{p}^{\prime}-\mathbf{q}\right)^{2}\left(2 m E-\mathbf{q}^{2}\right)} C_{A}\left[C_{F}\left(C_{A}^{2}-6 C_{A} C_{F}+8 C_{F}^{2}\right) 1 \otimes \overline{1}+\left(C_{A}^{2}-6 C_{F} C_{A}+12 C_{F}^{2}\right) T^{A} \otimes \bar{T}^{A}\right]$ \\
\hline iii & $\begin{array}{l}\frac{C_{A}}{16 \pi^{2}}\left[-\frac{1}{\epsilon^{2}} C_{A}\left[\left(C_{A}-2 C_{F}\right) C_{F}\left(f_{0}+f_{1}\right) 1 \otimes \overline{1}+\left(C_{A}-4 C_{F}\right) f_{1} T^{A} \otimes \bar{T}^{A}\right]+\right. \\
+\frac{1}{9 \epsilon}\left[C_{F}\left(C_{A}-2 C_{F}\right) 1 \otimes \overline{1}\left\{f_{0}\left[6 C_{A}+\left(2 C_{A}-12 C_{F}\right) \pi^{2}\right]-2 f_{1}\left[-3 C_{A}+\left(2 C_{A}-6 C_{F}\right) \pi^{2}\right]\right\}+\right. \\
\left.\left.+T^{A} \otimes \bar{T}^{A}\left\{12 C_{F}\left(2 C_{F}-C_{A}\right) \pi^{2} f_{0}+f_{1}\left[6 C_{A}\left(C_{A}-4 C_{F}\right)-4\left(C_{A}^{2}-4 C_{F} C_{A}+6 C_{F}^{2}\right) \pi^{2}\right]\right\}\right]\right]\end{array}$ \\
\hline $\mathrm{iv}+\mathrm{v}+\mathrm{vi}$ & $\begin{array}{l}\frac{C_{A}}{16 \pi^{2}}\left[\frac{1}{\epsilon^{2}} C_{A}\left[\left(C_{A}-2 C_{F}\right) C_{F}\left(f_{0}+f_{1}\right) 1 \otimes \overline{1}+\left(C_{A}-4 C_{F}\right) f_{1} T^{A} \otimes \bar{T}^{A}\right]+\right. \\
+\frac{1}{9 \epsilon}\left[C_{F}\left(C_{A}-2 C_{F}\right) 1 \otimes \overline{1}\left\{-5 f_{0}\left[6 C_{A}+\left(2 C_{A}-12 C_{F}\right) \pi^{2}\right]+10 f_{1}\left[-3 C_{A}+\left(2 C_{A}-6 C_{F}\right) \pi^{2}\right]-4 C_{A} f_{2}\left[12-2 \pi^{2}\right]\right\}\right. \\
+T^{A} \otimes \bar{T}^{A}\left\{60\left(C_{A}-2 C_{F}\right) C_{F} \pi^{2} f_{0}+f_{1}\left[-30 C_{A}\left(C_{A}-4 C_{F}\right)+20\left(C_{A}^{2}-4 C_{F} C_{A}+6 C_{F}^{2}\right) \pi^{2}\right]+\right. \\
\left.\left.\left.\quad+8 C_{A} f_{2}\left[-3 C_{A}+12 C_{F}+\left(2 C_{A}-2 C_{F}\right) \pi^{2}\right]\right\}\right]\right]\end{array}$ \\
\hline
\end{tabular}

TABLE II: UV divergent contributions before the q integration from three-loop diagrams with one potential and two ultrasoft loops as defined in table I. Subdivergences have been subtracted by one- and two-loop counterdiagrams. The soft three-momentum structure is (mainly) encoded in the $f_{i}$ functions of Eq. (13).

sum-operator counterterms $\delta \mathcal{V}_{k i}$. They take the generic form

$$
\delta \mathcal{V}_{k i}=A_{i} \frac{\alpha_{U}-\alpha_{S}}{\epsilon}+\tilde{A}_{i} \frac{\alpha_{U}^{2}-\alpha_{S}^{2}}{\epsilon^{2}}+B_{i} \frac{\alpha_{U}^{2}-\alpha_{S}^{2}}{\epsilon}
$$

In addition to Eq. (23) we included here, following Refs. [13] and [28], so-called soft pull-up terms at $\mathcal{O}\left(\alpha_{S}\right)$ and $\mathcal{O}\left(\alpha_{S}^{2}\right)$. They originate from the zerobin subtractions in corresponding amplitudes with one and two soft loops [28]. The ultrasoft one-loop coefficients $A_{i}$ were given in Ref. [13] and can be read off Eq. (23). They read in the color singlet channel

$$
A_{1}^{(s)}=-\frac{C_{A} C_{F}\left(C_{A}-2 C_{F}\right)}{3 \pi}, \quad A_{2}^{(s)}=\frac{C_{A} C_{F}\left(C_{A}-4 C_{F}\right)}{3 \pi}, \quad A_{3}^{(s)}=0 .
$$

For the anomalous dimension of the $\mathcal{V}_{k i}(\nu)$ to be finite, the relation

$$
\tilde{A}_{i}=-\frac{\beta_{0}}{8 \pi} A_{i}
$$

is a necessary condition. 


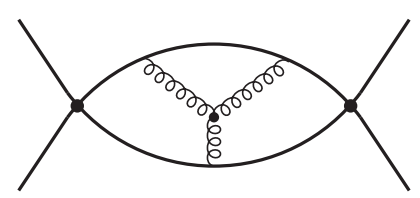

FIG. 4: Mercedes star type diagram contained in diagram class 9 (in table I). Together with the Mercedes star diagrams of class 10 it gives rise to the non-Abelian contribution (i+ii) in table II] The upside down graph is understood.

Summing all Abelian and non-Abelian contributions in table II except for contribution (i+ii), which we will discuss later, and projecting the result on the physical color singlet channel we find:

$$
\begin{aligned}
\mathrm{I}+\mathrm{II}+ & \mathrm{III}+\mathrm{IV}+\mathrm{V}+\mathrm{iii}+\mathrm{iv}+\mathrm{v}+\mathrm{vi}=C_{A}^{2}\left[\frac{1}{12 \epsilon^{2}}-\frac{7}{24 \epsilon}-\frac{\pi^{2}}{18 \epsilon}\right] \frac{i \alpha_{U}^{2}\left(\mathcal{V}_{c}^{(T)}\right)^{2} \mu_{S}^{4 \epsilon}}{m \pi^{2}} \\
& \times \int \frac{d^{d-1} q}{(2 \pi)^{d-1}}\left[C_{F}\left(C_{A}-2 C_{F}\right)\left(f_{0}+f_{1}+2 f_{2}\right)-C_{F}\left(C_{A}-4 C_{F}\right)\left(f_{1}+f_{2}\right)\right] \mathrm{P}_{\text {Singlet }} .
\end{aligned}
$$

The $\mathcal{O}\left(\alpha_{U}^{2} / \epsilon^{2}\right)$ terms of Eq. (24) and Eq. (28) satisfy Eq. (27), which provides an important cross-check of our calculation. From the respective $\mathcal{O}\left(\alpha_{U}^{2} / \epsilon\right)$ terms we determine the coefficients

$$
\begin{aligned}
& B_{1}^{(s)}=-\frac{C_{A}\left(C_{A}-2 C_{F}\right) C_{F}\left[C_{A}\left(47+6 \pi^{2}\right)-10 n_{f} T\right]}{108 \pi^{2}}, \\
& B_{2}^{(s)}=\frac{C_{A}\left(C_{A}-4 C_{F}\right) C_{F}\left[C_{A}\left(47+6 \pi^{2}\right)-10 n_{f} T\right]}{108 \pi^{2}} .
\end{aligned}
$$

The contribution (i+ii) in table is generated exclusively by those diagrams of class 9 and 10 which belong to the subclass with the Mercedes star topology shown in Fig. 4. All other diagrams of class 9 and 10 add up to zero. The result requires a separate discussion. It has already been computed in Ref. [23], but not considered there for numerical examinations. Although its effects are numerically tiny, they are interesting conceptually and worth to explain in some detail. Up to a color factor the term (i+ii) has the form

$$
\frac{\mathbf{q}^{2}}{48 \epsilon(\mathbf{q}-\mathbf{p})^{2}\left(\mathbf{p}^{\prime}-\mathbf{q}\right)^{2}\left(2 m E-\mathbf{q}^{2}\right)}
$$

If one carries out the renormalization procedure based on 5-loop current-current correlators instead of quark-antiquark scattering amplitudes, as realized in Ref. [16] for the computation of the 3-loop NNLL non-mixing contributions to the anomalous dimension of $c_{1}$, this term generates additional ultrasoft NLL terms in the counterterms of the $1 / \mathrm{m}^{2}$ potential coefficient $\mathcal{V}_{r}$ and of the coefficient of the $1 / \mathrm{m}^{2}$-suppressed S-wave current $c_{2}$. These contributions have not been considered in the literature before. They were also not contained in the results of Ref. 21] since they do not arise for scattering diagrams with equal external off-shellness, which was used for regulating IR divergences in Ref. [21]. Figure 5 shows a sketch of one of the 5-loop current-current correlator diagrams involved in the renormalization procedure following Ref. [16]. The divergences of these 5-loop diagrams have to be canceled by 3-loop current-current diagams without ultrasoft gluons, but with one of the potentials/currents 


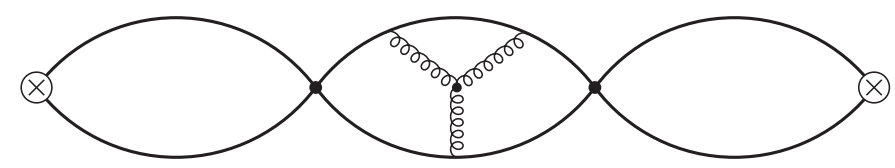

FIG. 5: 5-loop current-current correlator with a Mercedes star subdiagram. It is relevant in the current-current correlator based renormalization procedure associated with the divergent term (i+ii) in table II. In this approach one also has to take into account the 5-loop diagrams, where the Mercedes star is inserted into the left and right potential loop. The latter (partly) contribute to the renormalization of the $1 / m^{2}$-suppressed production/annihilation currents.

replaced by the respective $1 / \mathrm{m}^{2}$ suppressed counterterm. This approach yields an additional contribution to the counterterm $\delta \mathcal{V}_{r}^{(s)}$ to be added to the expression given in Ref. [21]. It results from insertions corresponding to the term (i+ii) in table II into a current-current correlator and reads

$$
\Delta \delta \mathcal{V}_{r}^{(s)}=2\left(4 \pi \alpha_{S}\right) \Delta B_{\mathbf{p}}^{(s)} ; \quad \Delta B_{\mathbf{p}}^{(s)}=\frac{C_{A} C_{F}^{2}}{24}
$$

Alternatively one might write Eq. (30) as

$$
-\frac{f_{0}+f_{1}}{96 \epsilon}+\frac{2 m E}{48 \epsilon(\mathbf{q}-\mathbf{p})^{2}\left(\mathbf{p}^{\prime}-\mathbf{q}\right)^{2}\left(2 m E-\mathbf{q}^{2}\right)}
$$

and directly absorb the ultrasoft divergence $\propto f_{0}+f_{1}$ into the counterterms $\delta \mathcal{V}_{k 3}$, while the counterterm $\delta \mathcal{V}_{r}^{(s)}$ of Ref. [21] and all other results in that reference remain unchanged. This leads to the coefficient

$$
B_{3}^{(s)}=\frac{C_{A} C_{F}^{3}}{24}
$$

The second term of Eq. (32) cannot contribute to the anomalous dimension of $c_{1}$, because the integrations over the soft momenta $\mathbf{q}, \mathbf{p}, \mathbf{p}^{\prime}$ give finite results, when the structure is inserted into a current-current correlator. It would rather contribute to the running of $c_{2}$, which becomes clear by rewriting its numerator as $2 m E=1 / 2\left(\mathbf{p}^{2}+\mathbf{p}^{\prime 2}\right)$ plus scaleless integrals (over $\mathbf{p}$ and $\mathbf{p}^{\prime}$ ). The remaining divergence could then be absorbed by the counterterms $\delta c_{2}$ of the $\mathbf{p}^{2} / m^{2}$-currents to the left and to the right of the current correlator.

The ambiguity in the renormalization procedure for the contribution $(\mathrm{i}+\mathrm{ii})$ is related to the freedom of performing field redefinitions and does not affect physical observables like the running of $c_{1}$. The contribution from (i+ii) to the $c_{1}$ evolution equation Eq. (2) is independent of whether we use the counterterm $\delta \mathcal{V}_{r}$ or $\delta \mathcal{V}_{k 3}$ to subtract the relevant divergences. As we will see below, $B_{\mathbf{p}}$ and $B_{3}$ enter the final expression for the NNLL anomalous dimension of $c_{1}$ in the combination $C_{F} B_{\mathbf{p}}+B_{3}$ (see footnote 7). Thus both approaches lead to the same result. Together with Eq. (29), Eq. (31) and (33) represent the main results of this work.

\section{SUMMARY OF RESULTS}

From Eqs. (19), (25) and (27) we obtain the anomalous dimensions

$$
\nu \frac{d}{d \nu} \mathcal{V}_{k i}=-\nu \frac{d}{d \nu} \delta \mathcal{V}_{k i}=2 A_{i}\left(2 \alpha_{U}-\alpha_{S}\right)+4 B_{i}\left(2 \alpha_{U}^{2}-\alpha_{S}^{2}\right) .
$$


To integrate Eq. (34) we can assume NLL running of $\alpha_{U}$ and $\alpha_{S}$ in the $A_{i}$ and LL running in the $B_{i}$ terms. Respecting the matching conditions $\mathcal{V}_{k i}(\nu=1)=0$, the solution of Eq. (34) at NLL order precision reads

$$
\mathcal{V}_{k i}^{\mathrm{NLL}}(\nu)=-\frac{4 \pi}{\beta_{0}} A_{i} \ln \frac{\alpha_{U}}{\alpha_{S}}+\left(\frac{\beta_{1}}{\beta_{0}^{2}} A_{i}-\frac{8 \pi}{\beta_{0}} B_{i}\right)\left(\alpha_{U}-\alpha_{S}\right),
$$

where $\beta_{0}=\frac{11}{3} C_{A}-\frac{4}{3} T n_{f}, \beta_{1}=\frac{34}{3} C_{A}^{2}-4 C_{F} T n_{f}-\frac{20}{3} C_{A} T n_{f}$ are the standard one- and twoloop coefficients of the QCD beta function. Eq. (35) is simple, because it only accounts for purely ultrasoft effects (plus the corresponding pull-up terms). We recall that the coefficients $\mathcal{V}_{k i}^{(s)}$ contribute to the NLL anomalous dimension of $c_{1}$ with an additional factor $\left[\mathcal{V}_{c}^{(T), \operatorname{LL}}(\nu)\right]^{2}$ according to the definition of the $\mathcal{O}_{k i}$ sum operators.

The corresponding expressions for the $1 / \mathrm{m}^{2}$ potentials are given by [21]

$$
\begin{aligned}
& \left(\mathcal{V}_{2}^{(s)}(\nu)\right)_{u s}^{\mathrm{NLL}}=\mathcal{V}_{c}^{(T), \mathrm{LL}}(\nu)\left[-\frac{4 \pi}{\beta_{0}} A_{\mathbf{k}}^{(s)} \ln \frac{\alpha_{U}}{\alpha_{S}}+\left(\frac{\beta_{1}}{\beta_{0}^{2}} A_{\mathbf{k}}^{(s)}-\frac{8 \pi}{\beta_{0}} B_{\mathbf{k}}^{(s)}\right)\left(\alpha_{U}-\alpha_{S}\right)\right], \\
& \left(\mathcal{V}_{r}^{(s)}(\nu)\right)_{u s}^{\mathrm{NLL}}=2 \mathcal{V}_{c}^{(T), \mathrm{LL}}(\nu)\left[-\frac{4 \pi}{\beta_{0}} A_{\mathbf{p}}^{(s)} \ln \frac{\alpha_{U}}{\alpha_{S}}+\left(\frac{\beta_{1}}{\beta_{0}^{2}} A_{\mathbf{p}}^{(s)}-\frac{8 \pi}{\beta_{0}} B_{\mathbf{p}}^{(s)}\right)\left(\alpha_{U}-\alpha_{S}\right)\right] .
\end{aligned}
$$

In this work the soft Coulomb factor $\mathcal{V}_{c}^{(T), \mathrm{LL}}(\nu)$ has not been included in the $1 / \mathrm{m}^{2}$ potential operator structure in Eq. (8), but appears directly in the Wilson coefficients $\mathcal{V}_{2, r}$ in Eqs. (36) and (37). The square brackets have the same form as Eq. (35) and represent the ultrasoft contributions (plus the corresponding pull-up terms) up to NLL order. In addition to Eqs. (36) and (37) there are also purely soft contributions to $\mathcal{V}_{2, r}$. They are fully known at LL order and can be found in Ref. [12].

Adopting the convention in Eq. (31), i.e. adding the extra contribution $\Delta B_{\mathbf{p}}^{(s)}$ to the singlet coefficient $B_{\mathbf{p}}^{(s)}$ determined in Ref. [21] $]^{6}$ instead of introducing an additional coefficient $B_{3}^{(s)}$, we can summarize the coefficients in Eqs. (35)-(37) as follows:

$$
\begin{aligned}
& {\left[\begin{array}{l}
A_{\mathbf{k}}^{(s)} \\
B_{\mathbf{k}}^{(s)}
\end{array}\right]=C_{F}\left(C_{A}-2 C_{F}\right)\left[\begin{array}{l}
A^{(s)} \\
B^{(s)}
\end{array}\right],} \\
& {\left[\begin{array}{l}
A_{\mathbf{p}}^{(s)} \\
B_{\mathbf{p}}^{(s)}
\end{array}\right]=-C_{A} C_{F}\left[\begin{array}{l}
A^{(s)} \\
B^{(s)}
\end{array}\right],} \\
& {\left[\begin{array}{l}
A_{1}^{(s)} \\
B_{1}^{(s)}
\end{array}\right]=-C_{A} C_{F}\left(C_{A}-2 C_{F}\right)\left[\begin{array}{l}
A^{(s)} \\
B^{(s)}
\end{array}\right],} \\
& {\left[\begin{array}{l}
A_{2}^{(s)} \\
B_{2}^{(s)}
\end{array}\right]=C_{A} C_{F}\left(C_{A}-4 C_{F}\right)\left[\begin{array}{l}
A^{(s)} \\
B^{(s)}
\end{array}\right],}
\end{aligned}
$$

where

$$
A^{(s)}=\frac{1}{3 \pi} \quad \text { and } \quad B^{(s)}=\frac{C_{A}\left(47+6 \pi^{2}\right)-10 n_{f} T}{108 \pi^{2}}
$$

\footnotetext{
${ }^{6}$ For the results in Ref. [21] the scheme choice of Eq. [33) was employed.
} 
This simple form is quite suggestive because the ultrasoft corrections to the color singlet Wilson coefficients of the $1 / m|\mathbf{k}|$ and the $1 / m^{2}$ potentials are universal up to color factors. This appears to be a quite non-trivial fact given the complexity and differences in the computations for the $1 / m^{2}$ and $1 / m|\mathbf{k}|$ potentials. We emphasize again, however, that the form of Eq. (38) is depending on the scheme choice associated with Eq. (31).

Using Eqs. (10) and (15) we can now write down the final expression for the ultrasoft contributions (including soft mixing and pull-up terms) to the effective color singlet $V_{k}$ potential in Eq. (3) up to NLL order. The Wilson coefficient

$$
\begin{aligned}
& \left(\mathcal{V}_{k, \mathrm{eff}}^{(s)}(\nu)\right)_{u s}^{\mathrm{NLL}}=\frac{\left[\mathcal{V}_{c}^{(T), \mathrm{LL}}(\nu)\right]^{2}}{8 \pi^{2}}\left[3 \mathcal{V}_{k 1}^{(s)}(\nu)+2 \mathcal{V}_{k 2}^{(1)}(\nu)\right]^{\mathrm{NLL}} \\
& \quad=-C_{A} C_{F}\left(C_{A}+2 C_{F}\right) \frac{\left[\mathcal{V}_{c}^{(T), \mathrm{LL}}(\nu)\right]^{2}}{8 \pi^{2}}\left[-\frac{4 \pi}{\beta_{0}} A^{(s)} \ln \frac{\alpha_{U}}{\alpha_{S}}+\left(\frac{\beta_{1}}{\beta_{0}^{2}} A^{(s)}-\frac{8 \pi}{\beta_{0}} B^{(s)}\right)\left(\alpha_{U}-\alpha_{S}\right)\right]
\end{aligned}
$$

appears in the NLL anomalous dimension of the Wilson coefficient $c_{1}(\nu)$ of the ${ }^{3} S_{1}$ heavy quark current in Eq. (2). The LL running of the Coulomb coefficient $\mathcal{V}_{c}^{T}(\nu)$ is purely soft and given by $\mathcal{V}_{c}^{(T), \mathrm{LL}}(\nu)=4 \pi \alpha_{S}$. Together with the ultrasoft NLL running of the $1 / \mathrm{m}^{2}$ potential coefficients $\mathcal{V}_{2, r}$ in Eqs. (36) and (37), Eq. (40) constitutes the NNLL ultrasoft mixing contributions to the anomalous dimension of $c_{1}$.

It is now straightforward to derive from Eq. (2) the complete two-loop ultrasoft part of the NNLL mixing contributions to the running of the ${ }^{3} S_{1}$ current coefficient $c_{1}$. We can parametrize the form of $c_{1}$ as [16]

$$
\ln \left[\frac{c_{1}(\nu)}{c_{1}(1)}\right]=\xi^{\mathrm{NLL}}(\nu)+\left(\xi_{\mathrm{m}}^{\mathrm{NNLL}}(\nu)+\xi_{\mathrm{nm}}^{\mathrm{NNLL}}(\nu)\right)+\ldots
$$

where $\xi^{\mathrm{NLL}}$ is the well known NLL order contribution [13, 14] and $\xi_{n m}^{\mathrm{NNLL}}$ is the NNLL non-mixing contribution determined in Ref. [16]. The ultrasoft part of the NNLL mixing contribution $\xi_{m}^{\mathrm{NNLL}}$ is new and reads

$$
\begin{aligned}
\xi_{\mathrm{m}, \text { usoft }}^{\mathrm{NNLL}}= & \frac{2 \pi \beta_{1}}{\beta_{0}^{3}} \tilde{A} \alpha_{s}^{2}(m)\left[-\frac{7}{4}+\frac{\pi^{2}}{6}+z\left(1-\ln \frac{z}{2-z}\right)+z^{2}\left(\frac{3}{4}-\frac{1}{2} \ln z\right)\right. \\
& \left.-\ln ^{2}\left(\frac{z}{2}\right)+\ln ^{2}\left(\frac{z}{2-z}\right)-2 \operatorname{Li}_{2}\left(\frac{z}{2}\right)\right] \\
+ & \frac{8 \pi^{2}}{\beta_{0}^{2}} \tilde{B} \alpha_{s}^{2}(m)\left[3-2 z-z^{2}-4 \ln (2-z)\right],
\end{aligned}
$$

where $^{7}$

$$
\begin{aligned}
& \tilde{A}=C_{F}\left(A_{\mathbf{k}}^{(s)}+2 A_{\mathbf{p}}^{(s)}\right)+3 A_{1}^{(s)}+2 A_{2}^{(s)}=-C_{F}\left(C_{A}+C_{F}\right)\left(C_{A}+2 C_{F}\right) A^{(s)}, \\
& \tilde{B}=C_{F}\left(B_{\mathbf{k}}^{(s)}+2 B_{\mathbf{p}}^{(s)}\right)+3 B_{1}^{(s)}+2 B_{2}^{(s)}=-C_{F}\left(C_{A}+C_{F}\right)\left(C_{A}+2 C_{F}\right) B^{(s)},
\end{aligned}
$$

\footnotetext{
${ }^{7}$ If one uses the counterterm $\delta \mathcal{V}_{k 3}$ to absorb the divergent contribution i+ii, the RHS of Eq. (40) reads $\left[\mathcal{V}_{c}^{(T), \mathrm{LL}}(\nu)\right]^{2} /\left(8 \pi^{2}\right)\left[3 \mathcal{V}_{k 1}^{(s)}(\nu)+2 \mathcal{V}_{k 2}^{(1)}(\nu)+2 \mathcal{V}_{k 3}^{(s)}(\nu)\right]^{\mathrm{NLL}}$ and $\tilde{B}=C_{F}\left(B_{\mathbf{k}}^{(s)}+2 B_{\mathbf{p}}^{(s)}\right)+3 B_{1}^{(s)}+2 B_{2}^{(s)}+2 B_{3}^{(s)}=$ $-C_{F}\left(C_{A}+C_{F}\right)\left(C_{A}+2 C_{F}\right) B^{(s)}$.
} 
with

$$
z \equiv\left(\frac{\alpha_{s}(m \nu)}{\alpha_{s}(m)}\right)^{\mathrm{LL}}=\left(1+\frac{\alpha_{s}(m) \beta_{0}}{2 \pi} \ln \nu\right)^{-1}
$$

\section{NUMERICAL DISCUSSION}
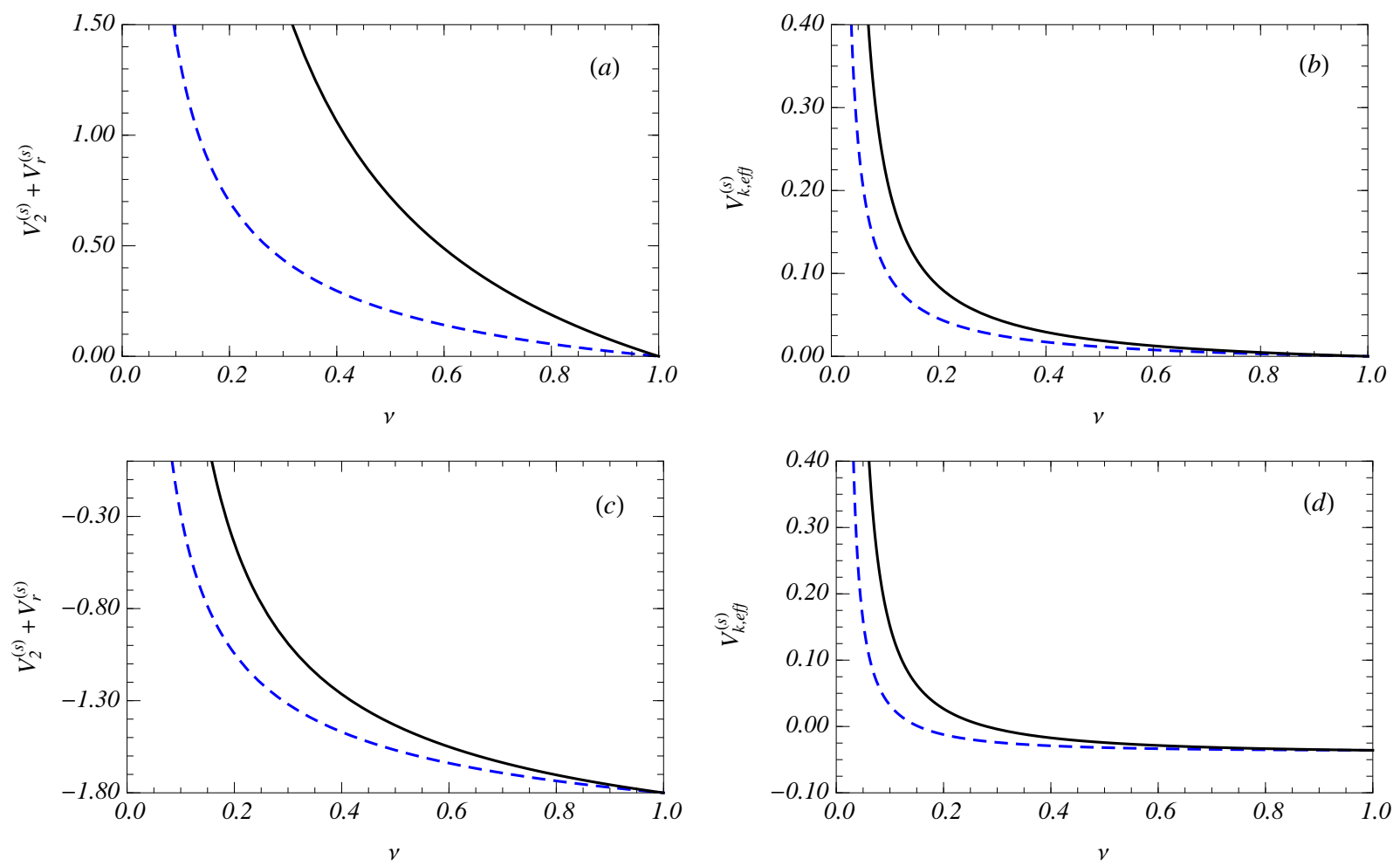

FIG. 6: Renormalization evolution in $\nu$ of $\mathcal{V}_{2}^{(s)}+\mathcal{V}_{r}^{(s)}$ and $\mathcal{V}_{k \text {,eff }}^{(s)}$. In panels a and b the ultrasoft LL (dashed lines) and LL+NLL evolution (solid lines) are shown assuming zero matching conditions at $\nu=1$. The curves include, however, the soft LL running of the factors $\mathcal{V}_{c}^{(T)}$ and $\left[\mathcal{V}_{c}^{(T)}\right]^{2}$ and pull-up terms as in Eqs. (36), (37) and (40). Panels c and d show the corresponding plots with the pure soft LL contributions and the corresponding matching conditions added to all curves.

In order to demonstrate the rather large size of the NLL order ultrasoft corrections to the anomalous dimensions we plot in Fig. 6 the evolution of the coefficients of the $1 / \mathrm{m}^{2}$ potentials $\left(\mathcal{V}_{2}^{(s)}+\mathcal{V}_{r}^{(s)}\right)$ and of the $1 / m|\mathbf{k}|$ potential $\mathcal{V}_{k, \text { eff }}^{(s)}$ at LL (dashed lines) and at NLL order (solid lines). These two sets of potentials are directly affected by ultrasoft effects. Since they appear explicitly in the NLL anomalous dimension of the ${ }^{3} S_{1}$ current, they constitute the most important ultrasoft mixing effects in the anomalous dimension of $c_{1}$, see Eq. (2). In panels $\mathrm{a}$ and $\mathrm{b}$ only the LL and NLL ultrasoft running (including soft mixing and pullup terms) according to Eqs. (36), (37) and (40) is displayed. For all curves zero matching conditions at $\nu=1$ has been adopted. For both $\mathcal{V}_{2}^{(s)}+\mathcal{V}_{r}^{(s)}$ and $\mathcal{V}_{k \text {,eff }}^{(s)}$ the ultrasoft NLL contributions are rather large and can even exceed the LL ones. The situation remains in 
Figs. 6r and d where we also include the full LL soft contributions and matching conditions from Ref. [12]. Due to the small prefactor $\sim \mathcal{V}_{c}^{(s)} /\left(16 \pi^{2}\right)$, the contribution from $\mathcal{V}_{2}^{(s)}+\mathcal{V}_{r}^{(s)}$ is relatively suppressed compared to the effect of $\mathcal{V}_{k \text {,eff }}$ in the evolution of $c_{1}$, Eq. (2). Therefore the ultrasoft NLL corrections to $\mathcal{V}_{k \text {,eff }}$ obtained in this work have a substantially larger impact on $c_{1}$ than those for $\mathcal{V}_{2, r}^{(s)}$ obtained in Ref. [21].

The interplay of the ultrasoft NLL evolution of the potential coefficients $\mathcal{V}_{2}, \mathcal{V}_{r}$ and $\mathcal{V}_{k \text {,eff }}$ in $c_{1}$ (see Eq. (21)) and the NNLL non-mixing contributions in the running of $c_{1}$ is examined for the case of top and bottom quark pairs in table III, which represents an update to table III of Ref. [16]. The values of $\ln c_{1}(\nu) / \ln c_{1}(1)$ at the NLL and NNLL level are given

\begin{tabular}{|c||c|c|c||c|c|c|}
\hline \multicolumn{1}{|c||}{} & \multicolumn{3}{c||}{$m=175 \mathrm{GeV}$} & \multicolumn{3}{c|}{$m=4.8 \mathrm{GeV}$} \\
\hline$\nu$ & $\xi^{\mathrm{NLL}}(\nu)$ & $\xi_{\mathrm{nm}}^{\mathrm{NNL}}(\nu)$ & $\xi_{\mathrm{nm}}^{\mathrm{NNLL}}(\nu)+\xi_{\mathrm{m}, \mathrm{us}}^{\mathrm{NNLL}}(\nu)$ & $\xi^{\mathrm{NLL}}(\nu)$ & $\xi_{\mathrm{nm}}^{\mathrm{NNL}}(\nu)$ & $\xi_{\mathrm{nm}}^{\mathrm{NNLL}}(\nu)+\xi_{\mathrm{m}, \mathrm{us}}^{\mathrm{NNLL}}(\nu)$ \\
\hline \hline 1.0 & 0.0000 & 0.0000 & 0.0000 & 0.0000 & 0.0000 & 0.0000 \\
\hline 0.9 & 0.0031 & 0.0018 & 0.0018 & 0.0127 & 0.0158 & 0.0153 \\
\hline 0.8 & 0.0066 & 0.0040 & 0.0039 & 0.0267 & 0.0360 & 0.0335 \\
\hline 0.7 & 0.0105 & 0.0066 & 0.0063 & 0.0419 & 0.0629 & 0.0555 \\
\hline 0.6 & 0.0150 & 0.0099 & 0.0091 & 0.0582 & 0.1008 & 0.0824 \\
\hline 0.5 & 0.0200 & 0.0142 & 0.0126 & 0.0743 & 0.1585 & 0.1154 \\
\hline 0.4 & 0.0259 & 0.0202 & 0.0171 & 0.0852 & 0.2584 & 0.1530 \\
\hline 0.3 & 0.0328 & 0.0294 & 0.0230 & 0.0689 & 0.4789 & 0.1671 \\
\hline 0.2 & 0.0401 & 0.0460 & 0.0313 & & & \\
\hline 0.1 & 0.0405 & 0.0916 & 0.0411 & & & \\
\hline
\end{tabular}

TABLE III: Numerical values for the contributions to the running of $\ln c_{1}(\nu) / \ln c_{1}(1)$ as defined in Eq. (411): $\xi^{\mathrm{NLL}}(\nu), \xi_{\mathrm{nm}}^{\mathrm{NNLL}}(\nu)$ and $\xi_{\mathrm{m}, \mathrm{Nu}}^{\mathrm{NNL}}(\nu)$. The values for $m$ are pole masses. The numbers are obtained by evaluation of the analytic results using one-loop running for $\alpha_{s}^{\mathrm{LL}}$ (in Eq. (45)) and taking $\alpha_{s}^{\left(n_{f}=5\right)}(175 \mathrm{GeV})=0.107$ and $\alpha_{s}^{\left(n_{f}=4\right)}(4.8 \mathrm{GeV})=0.216$ as input.

as a function of $\nu$ for the top quark $(m=175 \mathrm{GeV})$ and the bottom quark $(m=4.8 \mathrm{GeV})$ cases. The numbers in the third (sixth) column show the sum of the complete (soft and ultrasoft) NNLL non-mixing and ultrasoft mixing contributions. The latter terms were computed in this work. As a comparison we display in the second (fifth) column the nonmixing contributions alone. The first (fourth) column gives the number at the NLL order. As anticipated, we see that there is a substantial cancellation between the NNLL mixing and non-mixing contributions, particularly for small values of $\nu \sim \alpha_{s}$, where the NRQCD matrix elements are being evaluated. This behavior is insofar remarkable as the NLL nonmixing contributions of $c_{1}$ arise from 3-loop vertex diagrams while the mixing corrections are diagrammatically related to 4 -loop vertex diagrams. This demonstrates in a quite coercive way the importance of properly summing logarithms and of accounting for mixing effects in NRQCD predictions. The relation of mixing and non-mixing contributions to 3-loop and 4-loop vertex diagrams, respectively, also explains why the mixing contributions are so much smaller than the non-mixing ones close to the matching scale $\nu \sim 1$ where velocity logarithms are small.

A visual display of the results is given in Fig. 7 , where the evolution of $c_{1}(\nu) / c_{1}(1)$ is shown for top quark pair production close to threshold $(m=175 \mathrm{GeV})$. The blue dotted 
line represents the full NLL curve known before from Refs. [13, 14]. The red dashed line includes in addition the NNLL non-mixing contributions computed in Ref. [16] and exhibits a clear instability for $\nu \sim \alpha_{s} \sim 0.15$, where the low-energy matrix elements relevant for top threshold production have to be evaluated. This instability is the cause of the relatively large 6-10\% uncertainty in the normalization of the top pair threshold cross section [10, 19]. Finally, the black solid curve shows $c_{1}(\nu) / c_{1}(1)$ including the full NLL and NNLL non-mixing contributions as well as the ultrasoft NNLL mixing corrections finalized in this work. The stabilization of the renormalization group evolution for $\nu \sim \alpha_{s} \sim 0.15$ is obvious and will contribute to a reduction of the current uncertainties in RGI predictions for heavy quark pair threshold production. A detailed analysis of top and bottom quark production will be the subject of future publications.

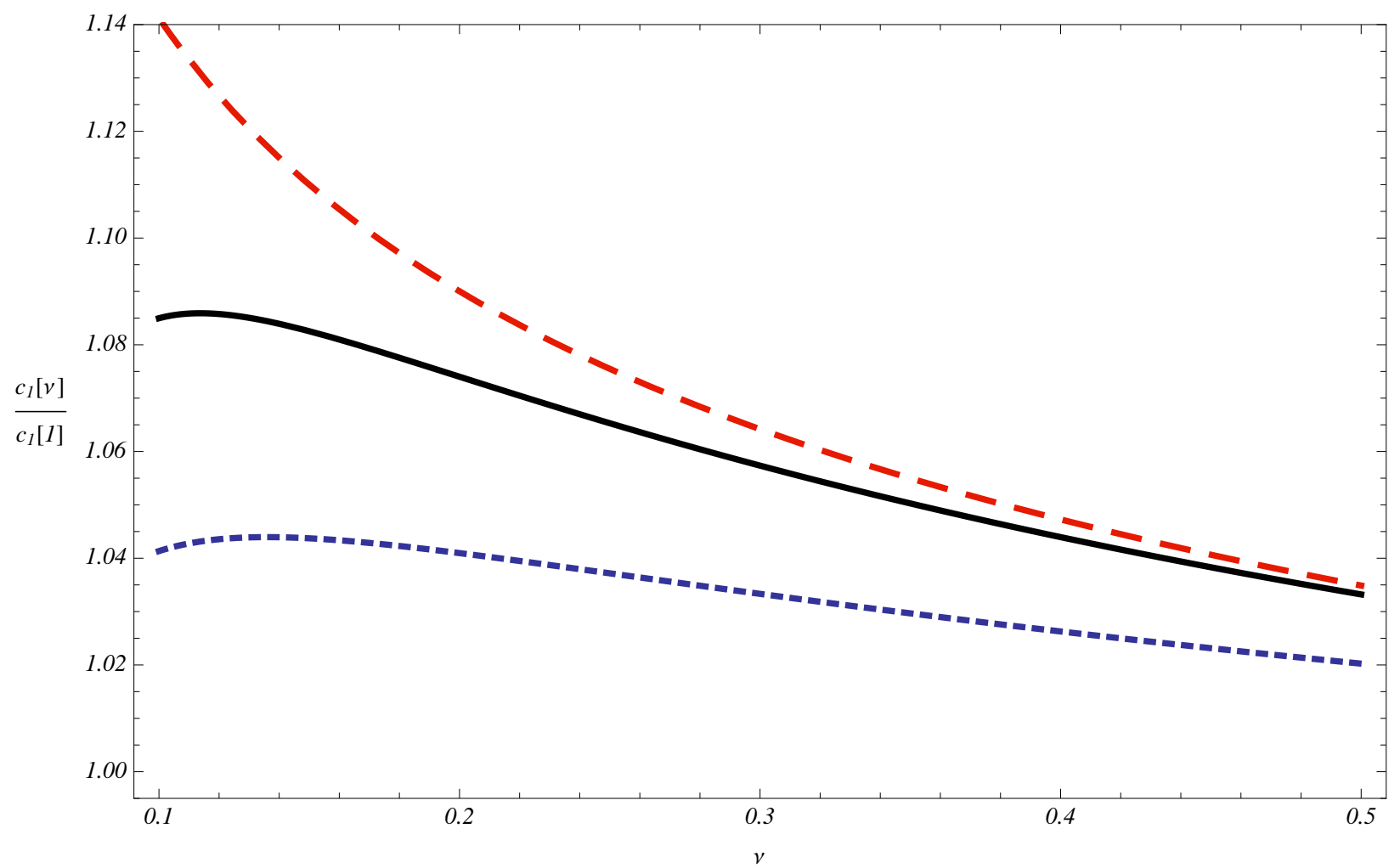

FIG. 7: Renormalization group evolution of the ${ }^{3} S_{1}$ current Wilson coefficient $c_{1}(\nu)$ normalized to $c_{1}(1)$ for the top quark case $(m=175 \mathrm{GeV})$. The dotted (blue) line represents the full NLL result $\exp \left[\xi^{\mathrm{NLL}}\right]$, the dashed (red) line includes in addition the NNLL non-mixing contributions, $\exp \left[\xi^{\mathrm{NLL}}+\xi_{\mathrm{nm}}^{\mathrm{NNLL}}\right]$. The solid (black) line accounts for the full NLL and NNLL non-mixing contribution as well as for the ultrasoft NNLL mixing corrections, $\exp \left[\xi^{\mathrm{NLL}}+\xi_{\mathrm{nm}}^{\mathrm{NNL}}+\xi_{\mathrm{m}, \mathrm{usoft}}^{\mathrm{NNL}}\right]$. The typical value for the velocity scaling parameter relevant for the evaluation of top threshold matrix elements is $\nu \sim \alpha_{s} \sim 0.15$. For the plot we have used $\alpha_{s}^{\left(n_{f}=5\right)}(175 \mathrm{GeV})=0.107$.

Nevertheless, one should not leave unmentioned that the NNLL corrections are obviously still quite large. In particular, scale variations obtained from the NLL order result around $\nu=0.15$ fail to give a reliable estimate of the perturbative uncertainty. For heavy quark threshold production such behavior is not an unfamiliar situation. For example, it is well known that the insertion of $\mathcal{O}\left(v^{2}\right)$ suppressed potentials and operators in NNLL order matrix 
elements leads to large corrections that cannot be anticipated from scale variation of the NLL results. In the case of the renormalization scale running of $c_{1}(\nu)$ the rather large NNLL shift arises from the lower order logarithms generated by the non-mixing contributions.

\section{CONCLUSION}

We have determined the two-loop ultrasoft corrections to the NLL order renormalization group equation of the $\mathcal{O}(v)$ vNRQCD $1 / m|\mathbf{k}|$ potentials. Combined with the previously known results for the $\mathcal{O}\left(v^{2}\right)$ potentials $\mathcal{V}_{r}^{(s)}(\nu)$ and $\mathcal{V}_{2}^{(s)}(\nu)$ determined in Ref. [21] our result completes the ultrasoft NNLL mixing part of the renormalization group evolution of the leading ${ }^{3} S_{1}$ current that describes heavy quark-antiquark pair production at threshold. Together with the previously known NNLL non-mixing corrections our results present the parametrically dominant contributions of the NNLL anomalous dimension of the leading ${ }^{3} S_{1}$ current and should allow for meaningful RGI predictions for heavy quark threshold production at the NNLL level.

Note added: While this paper was finalized we obtained a letter by Pineda [29] on the NLL ultrasoft renormalization group running of the spin-independent $\mathcal{O}(1 / m)$ and $\mathcal{O}\left(1 / m^{2}\right)$ potentials within the pNRQCD effective field theory framework. Including the soft pull-up terms, see e.g. Eq. (25), we find agreement with our results, i.e. the previously known ultrasoft NLL results for the $1 / m^{2}$ vNRQCD potentials $V_{2}^{(s)}$ and $V_{r}^{(s)}$ [21] together with the $1 / m|\mathbf{k}|$ potentials obtained in this work. In particular our results in Eqs. (36), (37) and (40) agree with the corresponding position space expressions $\delta V_{r, R G}^{(2)}\left(r ; \nu_{s}, \nu_{p}\right), \delta V_{p^{2}, R G}^{(2)}\left(r ; \nu_{s}, \nu_{p}\right)$ and $\delta V_{s, R G}^{(1)}\left(r ; \nu_{s}, \nu_{p}\right)$ of Ref. [29], respectively, upon transformation to momentum space and after imposing the correlation $\nu_{p}=\nu_{s}^{2} / m \equiv \mu_{U}\left(=m \nu^{2}\right)$ between the pNRQCD soft matching scale $\nu_{s}$ and the pNRQCD ultrasoft renormalization scale $\nu_{p}$. We remind the reader that the vNRQCD subtraction velocity $\nu$ is dimensionless whereas the pNRQCD scales $\nu_{p}$ and $\nu_{s}$ as well as the ultrasoft vNRQCD scale $\mu_{U}$ have the dimension of a mass.

The two-loop ultrasoft computations in our present work and in Refs. [21, 23] were more involved than the corresponding ones in Ref. [29] using pNRQCD. This is related to the simpler power counting of pNRQCD where soft fluctuations are decoupled from potential and ultrasoft ones. In the vNRQCD formulation used in our work soft, potential and ultrasoft fluctuations are contained in the theory which allows to systematically treat higher order mixing effects involving all three types of fluctuations (see Ref. [16] for such a computation). The equivalence of the results obtained in Ref. [29] and the ones in this work (and in Refs. [21, 23]) is technically non-trivial and indicates that the structural differences in the vNRQCD and pNRQCD formulations might not affect the description of purely ultrasoft effects.

\section{Acknowledgments}

This work was supported in part by the EU network contract MRTN-CT-2006-035482 (FLAVIAnet). The Feynman diagrams in this paper have been drawn using JaxoDraw [30]. 


\section{Appendix A: Feynman Rules of vNRQCD}

In this Appendix we list the vNRQCD momentum space Feynman rules in Feynman gauge, which are relevant for our calculation. Soft $\left( \pm \mathbf{p}, \pm \mathbf{p}^{\prime}\right)$ and ultrasoft momenta $\left(\vec{k}, \vec{k}_{i}\right)$ of the heavy (anti)quarks are understood to point into the direction of the positive energy flow indicated by little arrows on the heavy quark lines. If not explicitly indicated in the pic-

tures the default soft and ultrasoft momentum of a heavy (anti)quark is $\mathbf{p}$ and $\vec{k}$, respectively.

Propagators:

$$
\begin{array}{lcc}
\text { ultrasoft } A^{0} \text { gluon: } & \text { कoworo } & \frac{-i \delta^{A B}}{k^{2}+i \epsilon} \\
\text { ultrasoft A gluon: } & \text { कowor } & \frac{i \delta^{i j} \delta^{A B}}{k^{2}+i \epsilon} \\
\text { heavy (anti)quark: } & \frac{i}{k_{0}-\frac{\mathbf{p}^{2}}{2 m}+i \epsilon}
\end{array}
$$

Heavy quark kinetic insertions:

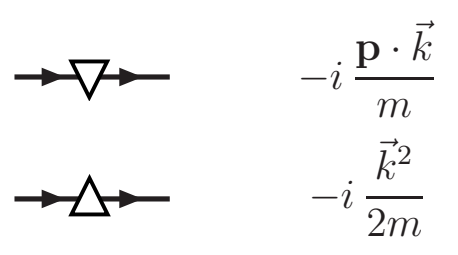

Heavy quark - ultrasoft gluon - vertices ${ }^{8}$ :
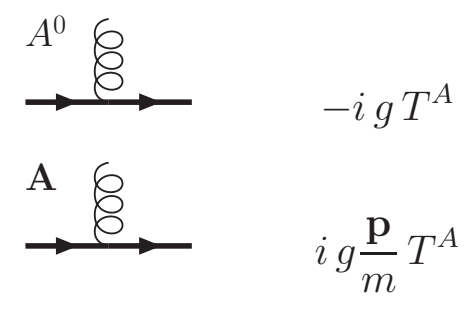

${ }^{8}$ For the respective gluon-antiquark vertices replace $T^{A} \rightarrow \bar{T}^{A}$. 
Triple ultrasoft gluon - vertices ${ }^{9}$ :
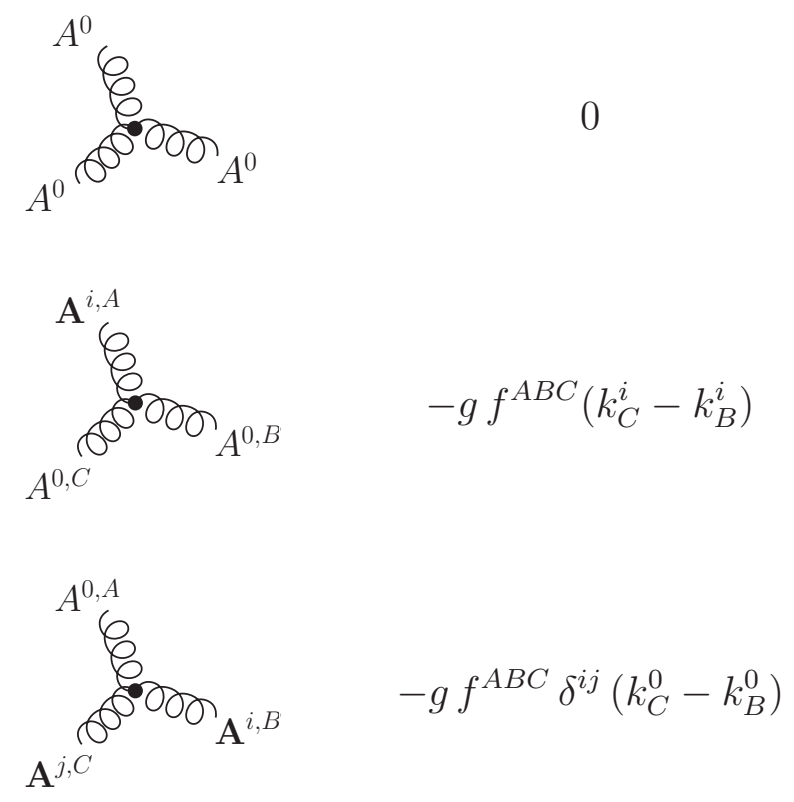

Heavy quark potential - vertices:

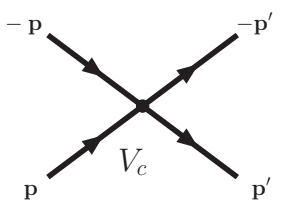

$$
\begin{aligned}
& \frac{-i \mathcal{V}_{c}^{(T)}}{\left(\mathbf{p}^{\prime}-\mathbf{p}\right)^{2}} T^{A} \otimes \bar{T}^{A} \\
& i \mathcal{V}_{c}^{(T)} T^{A} \otimes \bar{T}^{A} \frac{\left(\mathbf{p}^{\prime}-\mathbf{p}\right) \cdot\left[\left(\vec{k}_{4}-\vec{k}_{3}\right)-\left(\vec{k}_{2}-\vec{k}_{1}\right)\right]}{\left(\mathbf{p}^{\prime}-\mathbf{p}\right)^{4}} \\
& \left.-\frac{2\left[\left(\mathbf{p}^{\prime}-\mathbf{p}\right)\left(\vec{k}_{4}-\vec{k}_{3}\right)\right]^{2}+2\left[\left(\mathbf{p}^{\prime}-\mathbf{p}\right)\left(\vec{k}_{2}-\vec{k}_{1}\right)\right]^{2}}{\left(\mathbf{p}^{\prime}-\mathbf{p}\right)^{6}}\right] \\
& 2\left(\mathbf{p}^{\prime}-\mathbf{p}\right)^{4}
\end{aligned}
$$$$
\text { (1, }
$$$$
\text { p }
$$

Heavy quark potential - ultrasoft gluon - vertex:

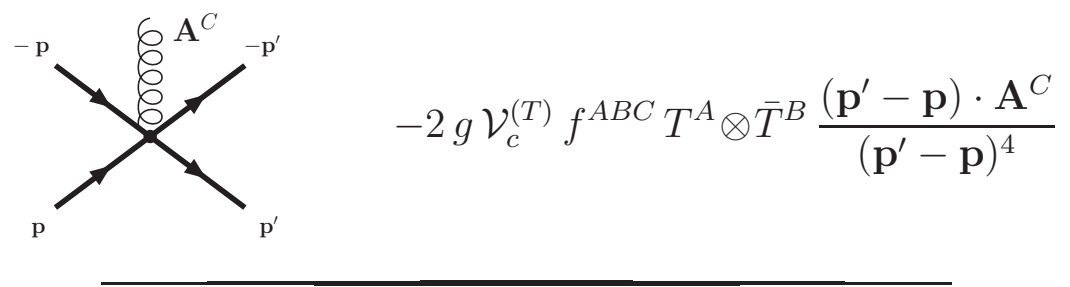

[1] J. A. Aguilar-Saavedra et al. [ECFA/DESY LC Physics Working Group], arXiv:hep-ph/0106315; T. Abe et al. [American Linear Collider Working Group], in

\footnotetext{
${ }^{9}$ All gluon four-momenta $k_{A, B, C}^{\mu}$ are incoming.
} 
Proc. of the APS/DPF/DPB Summer Study on the Future of Particle Physics (Snowmass 2001) ed. N. Graf, arXiv:hep-ex/0106057; A. Juste et al., arXiv:hep-ph/0601112.

[2] G. T. Bodwin, E. Braaten and G. P. Lepage, Phys. Rev. D 51, 1125 (1995) [Erratum-ibid. D 55, 5853 (1997)] arXiv:hep-ph/9407339].

[3] A. H. Hoang et al., Eur. Phys. J. directC 2, 1 (2000) arXiv:hep-ph/0001286.

[4] B. A. Kniehl, A. A. Penin, M. Steinhauser et al., Phys. Rev. Lett. 90, 212001 (2003). hep-ph/0210161.

[5] M. Beneke, Y. Kiyo, Phys. Lett. B668, 143-147 (2008). arXiv:0804.4004 [hep-ph]]; M. Beneke, Y. Kiyo, K. Schuller, Phys. Lett. B658, 222-229 (2008). [arXiv:0705.4518 [hep-ph]]; M. Beneke, Y. Kiyo, K. Schuller, Nucl. Phys. B714, 67-90 (2005). hep-ph/0501289.

[6] C. Anzai, Y. Kiyo, Y. Sumino, Phys. Rev. Lett. 104, 112003 (2010). arXiv:0911.4335 [hep$\mathrm{ph}]]$.

[7] A. V. Smirnov, V. A. Smirnov, M. Steinhauser, Phys. Rev. Lett. 104, 112002 (2010). arXiv:0911.4742 [hep-ph]].

[8] M. Beneke, Y. Kiyo, K. Schuller, PoS RADCOR2007, 051 (2007). arXiv:0801.3464 [hep$\mathrm{ph}]]$.

[9] A. H. Hoang, A. V. Manohar, I. W. Stewart and T. Teubner, Phys. Rev. Lett. 86, 1951 (2001) arXiv:hep-ph/0011254]; A. H. Hoang, A. V. Manohar, I. W. Stewart and T. Teubner, Phys. Rev. D 65, 014014 (2002) arXiv:hep-ph/0107144.

[10] A. Pineda and A. Signer, arXiv:hep-ph/0607239.

[11] M. Luke, A. Manohar and I. Rothstein, Phys. Rev. D61, 074025 (2000) arXiv:hep-ph/9910209.

[12] A.V. Manohar and I.W. Stewart, Phys. Rev. D 62, 014033 (2000) arXiv:hep-ph/9912226.

[13] A. H. Hoang and I. W. Stewart, Phys. Rev. D 67, 114020 (2003) arXiv:hep-ph/0209340.

[14] A. Pineda, Phys. Rev. D 66, 054022 (2002) arXiv:hep-ph/0110216.

[15] A. H. Hoang and P. Ruiz-Femenia, Phys. Rev. D 74, 114016 (2006) arXiv:hep-ph/0609151.

[16] A. H. Hoang, Phys. Rev. D 69, 034009 (2004) arXiv:hep-ph/0307376].

[17] A. Pineda, Phys. Rev. D 65, 074007 (2002) arXiv:hep-ph/0109117]; N. Brambilla, A. Pineda, J. Soto and A. Vairo, Phys. Rev. D 60, 091502 (1999) [arXiv:hep-ph/9903355.

[18] A. A. Penin, A. Pineda, V. A. Smirnov and M. Steinhauser, Phys. Lett. B 593, 124 (2004) arXiv:hep-ph/0403080.

[19] A. H. Hoang, Acta Phys. Polon. B 34, 4491 (2003) arXiv:hep-ph/0310301.

[20] A. A. Penin, A. Pineda, V. A. Smirnov and M. Steinhauser, Nucl. Phys. B 699, 183 (2004) arXiv:hep-ph/0406175.

[21] A. H. Hoang and M. Stahlhofen, Phys. Rev. D 75, 054025 (2007) arXiv:hep-ph/0611292.

[22] A. V. Manohar and I. W. Stewart, Phys. Rev. D 63, 054004 (2001) arXiv:hep-ph/0003107.

[23] M. Stahlhofen, PhD thesis, Technical University Munich, 2009, [ISBN 978-3-8381-0975-6], (http://nbn-resolving.de/urn/resolver.pl?urn:nbn:de:bvb:91-diss-20081230-680691-1-4).

[24] A. Pineda and J. Soto, Nucl. Phys. Proc. Suppl. 64, 428 (1998).

[25] M. Stahlhofen, Diploma Thesis, Technical University Munich, 2005.

[26] T. Huber and D. Maitre, Comput. Phys. Commun. 175, 122 (2006) arXiv:hep-ph/0507094.

[27] R. Mertig, M. Bohm and A. Denner, Comput. Phys. Commun. 64, 345 (1991).

[28] A. V. Manohar and I. W. Stewart, arXiv:hep-ph/0605001.

[29] A. Pineda, arXiv:1101.3269 [hep-ph].

[30] D. Binosi and L. Theussl, Comput. Phys. Commun. 161, 76 (2004) arXiv:hep-ph/0309015. 